\title{
Incentives and informal networks
}

Citation for published version (APA):

Fosco, C., \& Mengel, F. (2008). Incentives and informal networks. Maastricht University School of Business and Economics. METEOR Research Memorandum No. 022 https://doi.org/10.26481/umamet.2008022

Document status and date:

Published: 01/01/2008

DOI:

10.26481/umamet.2008022

Document Version:

Publisher's PDF, also known as Version of record

\section{Please check the document version of this publication:}

- A submitted manuscript is the version of the article upon submission and before peer-review. There can be important differences between the submitted version and the official published version of record.

People interested in the research are advised to contact the author for the final version of the publication, or visit the DOI to the publisher's website.

- The final author version and the galley proof are versions of the publication after peer review.

- The final published version features the final layout of the paper including the volume, issue and page numbers.

Link to publication

\footnotetext{
General rights rights.

- You may freely distribute the URL identifying the publication in the public portal. please follow below link for the End User Agreement:

www.umlib.nl/taverne-license

Take down policy

If you believe that this document breaches copyright please contact us at:

repository@maastrichtuniversity.nl

providing details and we will investigate your claim.
}

Copyright and moral rights for the publications made accessible in the public portal are retained by the authors and/or other copyright owners and it is a condition of accessing publications that users recognise and abide by the legal requirements associated with these

- Users may download and print one copy of any publication from the public portal for the purpose of private study or research.

- You may not further distribute the material or use it for any profit-making activity or commercial gain

If the publication is distributed under the terms of Article $25 \mathrm{fa}$ of the Dutch Copyright Act, indicated by the "Taverne" license above, 
Constanza Fosco, Friederike Mengel

Incentives and informal networks

$\mathrm{RM} / 08 / 022$

JEL code: D23, D82, J31

\section{METE@R}

Maastricht research school of Economics

of TEchnology and ORganizations

Universiteit Maastricht

Faculty of Economics and Business Administration P.O. Box 616

NL - 6200 MD Maastricht

phone : :+31433883830

fax : ++31433884873 


\title{
Incentives and informal networks*
}

\author{
Constanza Fosco ${ }^{\dagger}$ \\ Universidad Carlos III \\ Friederike Mengel ${ }^{\ddagger}$ \\ Maastricht University
}

\author{
July, 2008 \\ (first draft November 2007)
}

\begin{abstract}
We study a static model of principal-multiple agents with hidden actions (moral hazard) and local positive production externalities between agents. Agents interact through a given network of informal contacts, where links are interpreted as mutual advise or peer effect. Consequently, individual outputs will depend positively on own effort and nearest neighbors' efforts. As the principal only knows which kind of network prevails, but ignores the exact position of each agent, an additional problem of adverse selection arises. We restrict wages to be linear in individual output. Under risk neutrality and absence of limited liability constraints, the principal achieves (almost) the first best if either he observes efforts or knows the position of each agent in the informal network. The combination of hidden efforts and asymmetric information, though, yields inefficient results. Analyzing some paradigmatic network structures, we find that at the optimum the principal will offer a self selecting menu of contracts, although there may be less than perfect revelation. Which and how many types the principal will be able to distinguish depends heavily on the structure of the network and the strength of peer effects.
\end{abstract}

Keywords: Informal networks, incentive contracts.

JEL Classification: D23, D82, J31.

\footnotetext{
*This paper has benefitted from discussions with our supervisor Fernando Vega-Redondo. We also wish to thank Ani Guerdjikova, Karl Schlag and Marco van der Leij for helpful comments.

${ }^{\dagger}$ Corresponding author. Grupo Interdisciplinar de Sistemas Complejos (GISC)-Depto de Matemáticas, Universidad Carlos III de Madrid, Spain.e-mail: cfosco@math.uc3m.es.

${ }^{\ddagger}$ Department of Economics (AE1), Faculty of Economics and Business Administration, Maastricht University, PO Box 616,6200MD Maastricht, Netherlands. E-mail: F.Mengel@algec.unimaas.nl
} 


\section{Introduction}

Agents working in the same organization or firm are typically linked through some network of informal contacts. This informal network determines the flow of mutual advice and help between the agents and, as such, largely determines how agents behave within the firm. The importance of informal relations in shaping the incentives of workers to provide effort has long been recognized by studies in management and organizational science. Cross, Prusak and Parker (2002) from the Institute for Knowledge Based Organizations founded by IBM write "...how work gets accomplished is increasingly reliant on the health of informal networks. While often these groups are not found on any formal organizational chart, they frequently are the sources of both strategic and organizational success within an organization." Waldstrom (2001) summarizes the extensive literature in this field. ${ }^{1}$ Most of the research done in Economics is empirical. ${ }^{2}$ Theoretical models have also incorporated these concerns, although mostly have assumed global interactions, neglecting the "local" nature of peer effects. ${ }^{3}$ A thorough understanding of how informal networks shape incentives, though, is important for developing a viable theory of organizations.

In this paper we introduce informal networks in a standard principal - multiple agents framework. The set up is one where workers are paid by the contractor (the "principal") to produce some (observable) individual output. Workers and the principal are risk neutral and there are no limited liability constraints. The main channel through which the informal network affects the agent's incentives is that it creates effort externalities, meaning that an agent's marginal productivity is increased if her neighbors in the informal network exert higher effort. The size of the effort externality or strenght of peer effects is tempered by a fixed parameter. ${ }^{4}$ A basic difficulty of the principal is that - even if he might know the kind of informal network he is facing - he does not know the position of each agent in the network. The workers themselves though know their position and the position of others in the informal network much better than the principal. Thus there is a problem of adverse selection. A problem of moral hazard can arise in addition if the principal cannot observe efforts. Following Ballester, Calvó-Armengol and Zenou (2006), our assumptions about agents' production and cost functions imply that agents' first best and ex ante efficient efforts are proportional to their Bonacich (1987) centrality in the network. This centrality

\footnotetext{
${ }^{1}$ Other references include but are not limited to Krackhardt and Hanson (1993), Ruef, Aldrich and Carter (2003) or Di Maggio (2001).

${ }^{2}$ Ichino and Maggi (2000) show that workplace peers have a measurable impact on shirking and productivity; Azoulay and Zivin (2005) estimate the magnitude of knowledge spillovers generated by highly cited researchers in medicine; Mas and Moretti (2006) work tests Kandel and Laezer (1992) model (see footnote 4) and find evidence that social interactions affect individuals' productivity. Gould and Winter (2006) find evidence that peer effects may be caused by technological considerations (more precisely, they find that effort externalities may be related to the underlying production functions rather than to psychological considerations).; Bandiera, Barankay and Rasul (2007) find evidence of social concerns between workers and Shvydko (2007) finds evidence that such "social" peer effects might be stronger within small firms. Experimental evidence of pure social concerns has been found in Falk and Ichino (2006), among others.

${ }^{3}$ For instance, Kandel and Lazear (1992) analyze how peer pressure may create incentives which solve free riding problems within partnerships. Rotemberg (1994) develops a model of rational altruism, where the variables controlled by the workers (efforts) are strategically linked. Rob and Zemsky (1999) propose a dynamic principal-multiple agents model where agents are explicitly motivated by a combination of selfinterest and social concerns.

${ }^{4}$ Unlike in helping effort models with global interactions (e.g. Drago and Garvey (1998) or Itoh (1991)) we do not allow an agent to choose two different levels of effort (a private one and one directed to help others). Instead we assume that agents choose one effort level and that the externality is modulated by a fixed parameter.
} 
measure thus provides a summary of the private information any agent has.

We focus on a class of wages that are linear in observable individual outputs. Borrowing from Rob and Zemsky (1999) we call the marginal participation on individual output incentive intensity, as it assesses the extent to which the rewards of workers are linked to their measured performance. Whenever the incentive intensity is strictly positive, agents' expected utilities display positive externalities with respect to neighbors' efforts and the efforts of interacting agents are strategic complements.

Our results show that the structure of contracts depends critically on the shape of the informal network. If the principal has full information about the network, i.e. only faces a problem of moral hazard, he can achieve first-best allocations through personalized contracts. Under asymmetric information only (adverse selection), the principal optimally offers a self-selecting menu of contracts, with wages conditioned on outputs and efforts. He must pay informational rents to agents with a relative good position in the network, but these rents can be reduced arbitrarily. The reason is that for perfect revelation it is sufficient to offer to each "type" only a very small participation on her output conditional on that she exerts a certain observable level of effort. If the principal demands efficient efforts, he can get arbitrarily close to the first best as the incentive intensity gets smaller.

To summarize, when the principal faces the two problems separately, we find that (i) the first best can be (almost) achieved and (ii) this can be done through a set of contracts involving wages that are linear in individual productivity.

With respect to the generalized problem, we analyze two different situations. Firstly, we assume that there is only one contract available (Akerlof (1970), cf. Bolton, 2005), i.e. that the principal is ex ante constrained to offer the same contract to all agents. At the optimum, all agents but those with the lowest centrality will be paid in expectation participation rents. Through some simple examples, we find that the relation between the strength of peer effects and the optimal incentive intensity in the single contract is smooth but non-monotone. We also find that the principal obtains a fraction of first best expected profits which is decreasing in the strength of peer effects. For any given size of the effort externality, such relative inefficiency is positively correlated to the density, the variability of connections among agents and the average clustering of informal networks. ${ }^{5}$ Secondly, we allow the principal to offer a menu of self-selecting contracts. Of course such a wage scheme generally improves expected profits of the principal over the single contract. However, there might be institutional arrangements or administrative costs not modeled that prevent him to offer different contracts. That's why we think both problems are worthwhile studying.

When the principal offers different contracts, for any given strength of peer effects, he finds it optimal to offer discriminating wages with incentive intensities that are weakly increasing in the Bonacich centralities of agents. However, in some cases there may be less than perfect revelation as the principal pools some types and designs a common contract for them. Which and how many types are pooled depends on the particular network and the size of effort externality. In some networks the principal's profits are highest - relative to the first best case - if peer effects are either very weak or very strong. The relation between inefficiency and size of the effort externality is thus not generally monotone. Neither is it always the case that a high variability in agents connections prevents the principal from obtaining good results. Contrary to the case of single contracts, if peer effects are strong,

\footnotetext{
${ }^{5}$ We use the following definition for the clustering coefficient: the number of neighbors of any agent $i$ that are in turn connected relative to the number of different pairs of neighbors. Then, this is averaged over all agents.
} 
the principal can sometimes be better off if such variance is high.

Our model is closely related to the work of Ballester, Calvó-Armengol and Zenou (2006). ${ }^{6}$ They study a non-cooperative effort game in a network with local payoff complementarrities and show that Nash equilibrium effort choices are proportional to the Bonacich (1987) centrality of an agent in the network. Incentives in networks have also been studied in Kleinberg and Raghavan (2005), where agents provide information in large query networks and Bramoullé and Kranton (2007) where networked agents play a public good contribution game. To our knowledge our paper is unique in studying a "traditional" principal agent problem in networks. ${ }^{7}$

Naturally our paper is also related to vast literature on principal agent problems. Classical references include Mirrlees (1971, 1976), Grossman and Hart (1983), Guesnerie and Laffont (1984), Holmstrom (1979), Rogerson (1985) or Radner (1981). Most papers though analyze either only the problem of moral hazard or only the problem of adverse selection. The generalized problem, where both problems are present, has been studied by Riley (1985), Laffont and Tirole (1986), McAfee and McMillan (1987) or more recently Jullien, Salanié and Salanié (2007) and Faynzilberg and Kumar (2000). Principal-multiple-agents models have been proposed by Gupta and Romano (1998), Mookherjee (1984), Lockwood (2000) or Ishiguro and Itoh (2001), among others. Explicit teams have been analyzed by Andolfatto and Nosal (1997), Aoki (1994), Che and Yoo (2001), Groves (1973) or McAfee and McMillan (1991). Usually in these models agents are heterogeneous with respect to their skills or their costs of producing a certain level of effort. In our model the heterogeneity arises only from the different position the agents have in the network, provided that there are local positive production externalities. ${ }^{8}$ Abstracting from the informal network agents are homogeneous in skills or ability. This difference is not innocuous as our results show. Our paper is also one of the few in this literature to study the generalized problem with a finite and relatively large number of types.

The paper is organized as follows. In Section 2 we present the model. We analyze its results under observable efforts and full information (first best), and when each problem appears separately. In Section 3 we present all the results regarding optimal single contracts and self-selecting contracts in the generalized problem. Section 4 concludes. The proofs are relegated to an appendix.

\section{The Model}

\subsection{Description of the Model}

There is a finite set of agents $N=\{1,2, \ldots, n\}$ and one principal. Agents are identical with respect to ability, education and tasks and have the same labor status according to the formal chart of the organization. There exists, however, a hidden informal organization

\footnotetext{
${ }^{6}$ See also Ballester and Calvó-Armengol (2006), Calvó-Armengol and De Marti (2007) or Cabrales, Calvó-Armengol and Zenou (2007).

${ }^{7}$ In computer science principal-agent models have been proposed to overcome the free-riding behavior in peer-to-peer (P2P) systems. Feldman, Chuang, Stoica and Shenker (2005) study a problem of hidden actions where the network is a line. The "principal" are the end nodes, who provide incentives to the intermediate nodes.

${ }^{8}$ Lockwood (2000) proposes a multiple-agent framework where there are positive production externalities between agents, as in ours. The difference is that in his model all agents interact with each other and that they have differing cost-effort functions.
} 
shaped by the social network of informal relationships. In this network two agents are connected whenever they usually interact. Each link reflects positive peer effects that improve both individuals' productivity. Agents in general differ with respect to the number of agents they informally interact with, and this is the sole source of heterogeneity among them.

The pattern of informal interactions is given by a network $g$. A convenient representation of $g$ is the adjacency matrix $\mathbf{G}$, a symmetric $n \times n$ matrix with $g_{i j}=1(0)$ if link $i j$ is present (absent); by convention $g_{i i}=0$. The set $N_{i}(g)=\left\{j \in N: g_{i j}=1\right\}$ denotes the agents $i$ informally interacts with ("neighbors of $i$ "), and $k_{i}(g)=\left|N_{i}(g)\right|$ is the number of neighbors of $i$ or connectivity in the network $g$.

Each agent $i$ produces an output $y_{i} \in \mathbb{R}_{+}$by exerting an effort $a_{i} \in \mathbb{R}_{+}$. Peer effects take the form of local externalities in production, i.e.an effort $a_{i}$ simultaneously affects $i$ 's and $i$ 's neighbors' outputs. We assume the following explicit functions:

$$
\begin{aligned}
& y_{i}=x_{i}++\varepsilon_{i} \\
& x_{i}=\left(1+\beta \sum_{j \in N_{i}(g)} a_{j}\right) a_{i}
\end{aligned}
$$

with $\beta>0$ (positive externalities). Noise $\varepsilon_{i}$ represents an endogenous idiosyncratic shock with $E\left(\varepsilon_{i}\right)=0, E\left(\varepsilon_{i} \varepsilon_{j}\right)=0 \forall i \neq j$. It prevents the principal to know (ex post) efforts from observable outputs. Each agent $i$ bears the full cost of her effort; the monetary cost given by:

$$
c\left(a_{i}\right)=\frac{a_{i}^{2}}{2}
$$

The principal cares about total output $Y=\sum_{i \in N} y_{i}$ and compensates agents. Denote by $w_{i}$ the monetary wage offered to agent $i$. Principal and agents are risk neutral, ex post utility functions are respectively:

$$
U_{P}=\sum_{i \in N}\left(y_{i}-w_{i}\right) ; \quad U_{i}=w_{i}-\frac{a_{i}^{2}}{2}, i=1,2, \ldots, n
$$

Agent's reservation utility is $\underline{U}=0$ and there are no limited liability constraints. The risk neutrality of agents rules out insurance considerations. The absence of limited liability constraints implies that contracts are to be $e x$ ante individual rational, i.e. agents are willing to participate if they obtain in expectation at least so much utility than in their outside option.

The principal aims to provide incentives that induce each agent to exert some desired level of effort. The problem he faces is that efforts might not be observable and that he might have imperfect information about the informal network. In particular, we will assume that the principal knows which kind of network prevails, but he doesn't know the exact position of each agent. For instance, he might know that the network is a star, but not who is the hub. We also assume that informal relations are relatively stable and that agents have always perfect information about the whole network.

The timing of the model is as follows. First the principal designs the contracts and offers them to the agents. Contracts can be conditioned only on observable (and verifiable) variables. ${ }^{9}$ A contract determines the wage each agent will earn after production takes

\footnotetext{
${ }^{9} \mathrm{We}$ assume that when a variable is observable to the principal, it is also verifiable.
} 
place. We allow for two situations: (i) only one contract is available (single contract); (ii) the principal proposes a self-selecting menu of contracts. Once contracts are offered, all agents decide simultaneously whether to accept or not (or in case (ii) which contract to select). Provided that all agents accepted/selected a contract, they decide how much effort to exert. Effort choices are made non-cooperatively in the effort network subgame. Production takes place and contracts are fulfilled.

Note that given a set of bilateral contract offers, there may be multiple equilibria in the contract selection subgame (or contract acceptance game in case (i)). Whenever this occurs, we assume that all agents coordinate on the most efficient equilibrium. Under this assumption there is no loss of generality in restricting attention to contract offers such that every agent $i$ ends up accepting (or selecting) her contract (Bolton and Dewatripont, 2005); Accepted (or selected) contracts are always enforceable. We also assume that side-payments between agents are not allowed and that the renegotiation of contracts is not possible.

We restrict the set of possible wage functions to the set of wages of the form $w_{i}=$ $\gamma_{i}+\delta_{i} y_{i}$, with $\gamma_{i}, \delta_{i} \in \mathbb{R}, \delta_{i} \geq 0$. Some variations, such as $w_{i}=\gamma_{i}+\delta_{i}\left(y_{i}+\sum_{j \in N_{i}(g)} y_{j}\right)$ are also considered. ${ }^{10} \gamma_{i}$ and $\delta_{i}$ are taken as given by agent $i$. We call $\delta_{i}$ the incentive intensity. It measures the extent to which compensations are linked to observable performance. The fixed term $\gamma_{i}$, whenever negative, has the interpretation of a fixed fee agent $i$ has to pay to the principal before production takes place.

\subsection{Some Benchmark Cases}

\section{First Best}

In the "first best" situation efforts are observable and the principal is fully informed about the network. He asks each agent to exert the optimal effort $a_{i}^{*}$ that internalizes the local externalities and extracts all the surplus up to the participation constraints. Denote by $\overrightarrow{\mathbf{a}}=\left(a_{1}, a_{2}, \ldots, a_{n}\right)^{T}$ a vector of efforts, $\overrightarrow{\mathbf{1}}$ a $n \times 1$ vector of ones and $\mathbf{I}$ the $n^{\text {th }}$ order identity matrix. ${ }^{11}$ Recall that $\mathbf{G}$ is the adjacency matrix of a given network $g$; we define $\bar{\lambda}_{G}=\max \left\{\lambda_{G}\right\}$ to be the maximal eigenvalue of $\mathbf{G}$.

Assumption 1 (A1) For any particular network $g, \beta \in\left(0, \frac{1}{2 \bar{\lambda}_{G}}\right)$.

Definition (ex ante efficiency) The vector of efforts $\overrightarrow{\mathbf{a}}$ is (ex ante) efficient if $\overrightarrow{\mathbf{a}} \in$ $\arg \max W=E U_{P}+\sum_{i \in N} E U_{i}=\sum_{i \in N}\left(x_{i}-\frac{a_{i}^{2}}{2}\right)$.

Proposition 1 (first best) Assume A1. In the first best situation the principal offers to each agent $i, w_{i}^{*}=\frac{\left(a_{i}^{*}\right)^{2}}{2}$ iff $a_{i}=a_{i}^{*}$ and $w_{i}^{*}=0$ otherwise. The vector of optimal and efficient efforts is $\overrightarrow{\mathbf{a}}^{*}=(\mathbf{I}-2 \beta \mathbf{G})^{-1} \overrightarrow{\mathbf{1}}$. Expected utilities are $E U_{i}=0 \forall i, E U_{P}^{*}=$ $\frac{1}{2} \overrightarrow{\mathbf{1}}^{T}(\mathbf{I}-2 \beta \mathbf{G})^{-1} \overrightarrow{\mathbf{1}}$. Efforts and $E U_{P}^{*}$ are increasing and convex functions of $\beta$.

Proof. In the Appendix.

Following Ballester, Calvó-Armengol and Zenou (2006) it is easy to see that first best efforts are proportional (in our case equivalent) to the non-weighted Bonacich centrality with parameter $2 \beta$ of each agent in the network. Indeed (see their Definition 1), provided

\footnotetext{
${ }^{10}$ Note that the last formulation is only possible if the principal knows exactly the network.

${ }^{11}$ Throughout the paper, vectors are column vectors. Supraindex $T$ denotes transpose.
} 
that $(\mathbf{I}-2 \beta \mathbf{G})^{-1}$ is well defined and nonnegative, the vector of Bonacich centralities of parameter $2 \beta$ in $g$ is $\overrightarrow{\mathbf{b}}(g, 2 \beta)=(\mathbf{I}-2 \beta \mathbf{G})^{-1} \overrightarrow{\mathbf{1}}$. Assumption 1 is necessary and sufficient for $(\mathbf{I}-2 \beta \mathbf{G})$ to be inverse-positive. The Bonacich (1987) centrality basically measures how many paths in the network start at a particular node $i$. The relative weight of longer paths is scaled down by $2 \beta$. A 1 amounts to saying that the payoff complementariness are not too important compared with the concavity due to own effort. This assumption seems particularly sensible, as we deal with informal networks within an organization. Below though we will see that even these relatively small network effects can have large effects on the agent's incentives and on the optimal contract.

\section{Moral hazard}

Suppose now that the principal is fully informed about the interaction structure but cannot observe efforts. Then we face a pure problem of moral hazard. We show that there are (at least) two contracts through which first best efforts can be enforced. In both, wages are personalized to each agent. The difference is that while in the first, wages are only a function of agent's $i$ product, in the second they depend on neighbors' products as well. Intuitively, the principal can induce agents to internalize local externalities either through the incentive intensity $\delta_{i}$ or (more directly) by giving them marginal participation on neighbors' outputs.

Proposition 2 (moral hazard) Assume A1 and that there is only a moral hazard problem. Then contracts which take one of these alternative forms,

$$
\begin{aligned}
& w_{i}=\gamma_{i}+\delta_{i} y_{i}, \text { with } \delta_{i}=1+\frac{\beta \sum_{j \in N_{i}(g)} a_{j}^{*}}{1+\beta \sum_{j \in N_{i}(g)} a_{j}^{*}}>1 \text { and } \gamma_{i}=-\delta_{i} x_{i}^{*}+\frac{\left(a_{i}^{*}\right)^{2}}{2} ; \text { or } \\
& w_{i}=\gamma_{i}+\delta_{i}\left(y_{i}+\sum_{j \in N_{i}(g)} y_{j}\right), \text { with } \delta_{i}=1 \text { and } \gamma_{i}=-\left(x_{i}^{*}+\sum_{j \in N_{i}(g)} x_{j}^{*}\right)+\frac{\left(a_{i}^{*}\right)^{2}}{2},
\end{aligned}
$$

$\forall i \in N$ allow the principal to attain first best (efficient) allocations.

Proof. In the Appendix.

Remark 1 The fixed terms $\gamma_{i}$ here have a double role. They allow the principal to extract all the surplus and at the same time guarantee that the allocation of total output is feasible in expected terms.

Remark 2 Note that although both type of contracts yield the same outcome, the second is more consistent with a setting where the principal only observes total output. Indeed, it is easy to see that analogous results could be found if personalized wages of the form $w_{i}=\gamma_{i}+\delta_{i} Y$ were offered $\left(Y=\sum_{j \in N} y_{j}\right){ }^{12}$

\footnotetext{
${ }^{12}$ Best response functions would be the same as in case (ii) because even if agents are paid as a function of all other agents' outputs, they would consider marginal incentives on $y_{i}+\sum_{j \in N_{i}} y_{j}$. Hence with $\delta_{i}=1$ best first efforts can be induced. Naturally, fixed terms $\gamma_{i}$ would be different: $\gamma_{i}=-X^{*}+\frac{\left(a_{i}^{*}\right)^{2}}{2}$, where $X^{*}$ is total expected output (when all agents exert first best efforts).
} 
Even if the principal doesn't observe efforts, the perfect information about the network is sufficient to estimate expected first best efforts and outputs. $\delta_{i}$ and $\gamma_{i}$ are parameters taken as given by the agents, who subsequently choose non-cooperatively their effort levels. The principal then induces Nash equilibrium efforts which coincide with first best efforts. This is possible essentially because agents are risk-neutral and the principal need not to insure them for their participation. Contracts similar to the second type can be found in McAfee and McMillan (1991) applied to teams. In the following we will focus uniquely on individual incentives.

\section{Adverse selection}

The problem of adverse selection arises if the principal observes efforts but has partial information about the network. In particular, we assume that the principal only knows which kind of network prevails, but doesn't know the exact position of agents. As first best efforts are proportional to the vector of non-weighted Bonacich centralities in the network $g$, this measure can be considered as the "type" of agent $i$. As we mentioned, provided that $1>2 \beta \bar{\lambda}_{G}, \overrightarrow{\mathbf{a}}^{*}=\overrightarrow{\mathbf{b}}(g, 2 \beta)=(\mathbf{I}-2 \beta \mathbf{G})^{-1} \overrightarrow{\mathbf{1}}$ is the $n \times 1$ vector of Bonacich (non-weighted) centrality of parameter $2 \beta$ in the network $g$ and $b_{i}(g, 2 \beta)$ is its $i^{\text {th }}$ element, the Bonacich index of agent $i$. Suppose that there are $r \leq n$ different centralities. We partition the set of agents $N$ into subsets $N(t)$ of agents of the same type: $N(t)=\left\{i \in N: b_{i}(g, 2 \beta)=b_{t}(g, 2 \beta)\right\}, N(t) \cap N\left(t^{\prime}\right)=\varnothing \forall t \neq t^{\prime}, \underset{t=1}{r} N(t)=N . T$ denotes the set of types $t, t=1, \ldots, r$ and without loss of generality, we assume that indexes are upward ordered, i.e. $t<t^{\prime}$ if and only if $b_{t}(g, 2 \beta)<b_{t^{\prime}}(g, 2 \beta)$. For instance, in a line of five (5) agents, there are three types (for any $\beta$ satisfying A1). End nodes are of the lowest type, while the agent in the middle would be the highest type.

In this context, wages can depend on efforts, outputs or both observable variables. We want to stress, however, that if the principal proposes a menu of self-selecting contracts, wages must necessarily depend on outputs. Wages depending only on efforts are never incentive compatible - unless the network is $k$-regular. ${ }^{13}$ The intuition for this is very simple. In our setup the type of an agent is uniquely determined by her position in the network. The different marginal productivities arise only from the positive effort externalities. Agents $d o$ not differ in their cost-functions for exerting effort. But this of course immediately implies, that if the principal pays only according to effort, then all types will choose the same contract and exert the same effort.

Proposition 3 (adverse selection - $w_{i}\left(a_{i}\right)$ ) Assume A1 and that there is only adverse selection. If wages depend only on efforts, then the optimal contract is a pooling contract: $w=\frac{\widetilde{a}^{2}}{2}$, with $\widetilde{a}=\frac{1}{1-2 \beta \bar{k}} ; E U_{i}=0 \forall i \in N ; \widetilde{E U}_{P}=n \frac{1}{2} \frac{1}{1-2 \beta \bar{k}}$. If the network is $k$-regular, the first best is achieved $\left(\widetilde{a}=a^{*}, \widetilde{E U}_{P}=E U_{P}^{*}\right)$, otherwise $\widetilde{E U}_{P}<E U_{P}^{*}$.

Proof. In the Appendix.

The asymmetry of information is an obstacle for the principal as long as the network is not regular. If the network is $k$-regular, the principal knows the common connectivity $k$ and can easily enforce first best efforts with a single contract, even if efforts are not observable, as we will see in Section 3.

\footnotetext{
${ }^{13} g$ is a $k$-regular network $\Longleftrightarrow k_{i}(g)=k \forall i \in N$.
} 
Suppose now that the principal offers wages depending on both observable variables. ${ }^{14}$ A contract intended to agents of type $t$ prescribes a given level of effort and the expected wage associated to that effort. Therefore, the principal takes into account the participation and incentive compatibility constraints with respect to the contract choice, but effort levels are not constrained to be part of a Nash equilibrium of an effort subgame. Thus when any agent deviates the rest of players are still choosing the "right" contract and the "right" effort. ${ }^{15}$ In this sense the principal's problem is isomorphic to a problem with one agent that can be of different types. Following standard arguments, the principal will have to pay informational rents, hence the first best cannot be achieved. However, as we show below, under our assumptions the principal can come arbitrarily close to the first best. Denote by $x_{t}^{*}$ the expected output of type $t$ in the first best situation, and $\widetilde{x}_{t \rightarrow t-1}$ the expected product of type $t$ when exerting the effort corresponding to type $t-1$, given that everyone else exerts $a_{t^{\prime}}^{*}$.

Proposition 4 (adverse selection - $w_{i}\left(a_{i}, y_{i}\right)$ ) Assume A1 and that there is only adverse selection. If the principal offers contracts $C_{T}=\left\{\left(\gamma_{t}, \delta_{t}\right), a_{t}^{*}\right\}_{t=1, \ldots, r}$; s.t. $w_{t(i)}=$ $\gamma_{t}+\delta_{t} y_{i}$ iff $a_{i}=a_{t}^{*}$, with $\delta_{t}=\varepsilon>0 \forall t, \gamma_{1}=-\varepsilon x_{1}^{*}+\frac{\left(a_{1}^{*}\right)^{2}}{2}$, and $\gamma_{t}=\gamma_{t-1}-\varepsilon\left(x_{t}^{*}-\right.$ $\left.\widetilde{x}_{t \rightarrow t-1}\right)+\frac{\left(a_{t}^{*}\right)^{2}-\left(a_{t-1}^{*}\right)^{2}}{2} \forall t \geq 2$; he will be able to come arbitrarily close to first best expected utility by choosing $\varepsilon$ arbitrarily small.

Proof. In the Appendix.

Remark 3 There are several similar contracts, we only propose one. What is needed is that wages for $t \geq 2$ depend on outputs and that incentive intensities $\delta_{t}$ are arbitrarily small. $\delta_{t}$ could be also different.

The intuition is simple. As long as wages depend on outputs, the principal can induce agents to "reveal" their types. The value of the fixed terms $\gamma_{t}$ are such that any agent of type $t$ is indifferent between choosing contract $c_{t}$ and $c_{t-1}$, as is standard in the literature. In other words, local downward incentive compatibility conditions are binding. Given this, no agent will have incentives to imitate other type, and agents with type $t \geq 2$ obtain informational rents. However, such rents can be reduced arbitrarily by setting the incentive intensity $\varepsilon$ very small, as for any $\varepsilon$ contracts are incentive compatible. What is crucial for this result is that the principal observes efforts and outputs (thus contracts can depend on both) and that agents are risk neutral. Interestingly, if there are only two types, as in the star, the first best can be exactly achieved. The principal would offer two contracts, $\left.c_{1}=\left(\left(\gamma_{1}, \delta_{1}\right), a_{1}\right)=\left(\left(\frac{\left(a_{1}^{*}\right)^{2}}{2}, 0\right), a_{1}^{*}\right)\right) ; c_{2}=\left(\left(\gamma_{2}, \delta_{2}\right), a_{2}\right)=\left(\left(-\delta_{2} x_{2}^{*}+\frac{\left(a_{2}^{*}\right)^{2}}{2}, \delta_{2}\right), a_{2}^{*}\right)$ and there is no need to pay informational rent to the highest type. ${ }^{16}$

\footnotetext{
${ }^{14}$ Clearly, with wages only conditioned on outputs, the solution would coincide with that of the general problem, Section 3. With such contracts, the principal would be throwing away important information he has, namely the observable levels of effort.

${ }^{15}$ When such deviation occurs, ex post wages of other agents can be affected because their output may be different, but their efforts do not change as in the general case.

${ }^{16}$ Use the same notation as in the proof of proposition 4. On the equilibrium path, expected outputs are: $x_{1}^{*}=\Phi_{1}^{*} a_{1}^{*}$ and $x_{2}^{*}=\Phi_{2}^{*} a_{2}^{*}$ with $\Phi_{2}^{*} \geq \Phi_{1}^{*}$ and $a_{2}^{*} \geq a_{1}^{*}$. If an agent of type $t=1,2$ deviates to $t^{\prime}=2,1, \widetilde{x}_{t \rightarrow t^{\prime}}=\Phi_{t}^{*} a_{t^{\prime}}^{*}$. Thus, if any agent chooses the right contract, $E U_{1}=\gamma_{1}-\frac{\left(a_{1}^{*}\right)^{2}}{2}=0, E U_{2}=$ $\gamma_{2}+\delta_{2} x_{2}^{*}-\frac{\left(a_{2}^{*}\right)^{2}}{2}=0$. If type 1 imitates type $2, E U_{1 \rightarrow 2}=\gamma_{2}+\delta_{2} \Phi_{1}^{*} a_{2}^{*}-\frac{\left(a_{2}^{*}\right)^{2}}{2}=-\delta_{2}\left(\Phi_{2}^{*}-\Phi_{1}^{*}\right) a_{2}^{*}<0$, while type 2 is indifferent: $E U_{2 \rightarrow 1}=\gamma_{1}-\frac{\left(a_{1}^{*}\right)^{2}}{2}=0$. It is easy to see that $E U_{P, A I}=E U_{P}^{*}$. These results are independent of the precise value of $\delta_{2}$.
} 


\section{Hidden efforts under asymmetric information}

Provided that agents are risk-neutral, inefficiency arises primarily due to the asymmetry of information, although the principal can get approximately the first best would he observe efforts. With hidden efforts and asymmetric information, contracts as proposed in Prop. 4 are not available, as wages cannot depend on efforts.

Still - also in the general problem - in any $k$-regular the first best efforts can be implemented without additional costs with a single (pooling) contract (see Prop. 6 below). What happens if the network is not regular? Interestingly, a "small" amount of heterogeneity suffices to yield very different results. Consider for example a circle network. It is regular and the principal is able to implement first best efforts. Now assume that a pair of agents in the circle are not linked. The network is now a line or chain and from the principal's viewpoint there are $\lceil n / 2\rceil$ "types" of agents. As we show below, there is no single contract $(\gamma, \delta)$ that allows the principal to implement first best efforts.

Remember that the general problem has three stages. First the principal proposes (i) either a menu of contracts or (ii) a single contract. As we already mentioned, the set of contracts $C_{T}$ is restricted to wages of the form $w\left(t, y_{i}\right)=\gamma_{t}+\delta_{t} y_{i}$. A contract $c_{t}$ for individuals of type $t$ (i.e. Bonacich centrality $\left.b_{t}\right)$ is defined by the pair $\left(\gamma_{t}, \delta_{t}\right)$. A single contract is of course independent of $t$. Then agents simultaneously select (or accept) a contract and finally they make their effort choices non-cooperatively. The principal's problem is thus constrained by both the contract choice subgame and the effort choice subgames. We solve the model by backward induction starting with the effort choice subgame.

Effort network subgame: Our first result concerns the existence and uniqueness of the Nash equilibrium in these subgames where we rely heavily on previous results from Ballester, Calvó-Armengol and Zenou (2006). Suppose that the principal has offered a menu of contracts, i.e. a set of pairs $\left(\gamma_{t}, \delta_{t}\right)$, from which in the intermediate subgame each agent chooses one. Agents can choose from any particular profile of incentive intensities $\overrightarrow{\mathbf{d}}=\left(\delta_{1}, \ldots, \delta_{n}\right)^{T}$, with each $\delta_{i} \in\left\{\delta_{t}\right\}_{t=1, \ldots, r}$. Different combinations induce different network effort subgames. Denote by $\bar{\delta}=\max \left\{\delta_{t}\right\}$ the maximum incentive intensity offered by the principal and let $\mathbf{D}$ be a diagonal matrix s.t. $\operatorname{diag}(\mathbf{D})=\overrightarrow{\mathbf{d}}$.

Proposition 5 (effort network subgame) Given any particular $g$ and $\beta$ satisfying A1. If $\bar{\delta} \in\left(0, \frac{1}{\beta \bar{\lambda}_{G}}\right)$ then an interior Nash equilibrium in the network effort subgame (in pure strategies) always exists and is unique for any profile $\overrightarrow{\mathbf{d}}$ chosen by the agents. For each $\overrightarrow{\mathbf{d}}$, the equilibrium effort profile is given by $\overrightarrow{\mathbf{a}}=(\mathbf{I}-\beta \mathbf{D G})^{-1} \overrightarrow{\mathbf{d}}$. Given two different profiles $\overrightarrow{\mathbf{d}}$ and $\overrightarrow{\widetilde{\mathbf{d}}}: \overrightarrow{\widetilde{\mathbf{d}}} \supsetneqq \overrightarrow{\mathbf{d}} \Longrightarrow \overrightarrow{\overrightarrow{\mathbf{a}}}>\overrightarrow{\mathbf{a}}$. Effort $\widehat{a}_{i}$ is strictly convex in $\delta_{i}$.

Proof. In the Appendix.

Efforts are proportional to the weighted Bonacich centrality of the agents. Given the network $g$ and the particular profile $\overrightarrow{\mathbf{d}}$, the vector of weighted Bonacich centralities is $\overrightarrow{\mathbf{b}}_{\mathbf{d}}=$ $(\mathbf{I}-\beta \mathbf{D G})^{-1} \overrightarrow{\mathbf{d}}$. Proposition 5 states a sufficient condition under which the equilibrium effort profile is interior and unique for any $\overrightarrow{\mathbf{d}}$ chosen in the asymmetric information stage.

Equilibrium efforts increase along with the incentive intensities $\overrightarrow{\mathbf{d}}$. This implies that when any agent is either offered or chooses a higher $\delta_{i}$, not only she but also all the agents connected to her through some path will increase their efforts etc.... This "contagion" effect is modulated by $\beta$, the incentive intensity and the distance between any two agents. 
Fixing the others' marginal participations, the effort of agent $i$ can ex ante be interpreted as a continuous, increasing and strictly convex function of $\delta_{i}{ }^{17}$

The asymmetric information stage: The principal wants to induce each agent of type $t$ to choose the contract $c_{t}=\left(\gamma_{t}, \delta_{t}\right)$. All agents must participate and the contracts chosen must be part of a Nash equilibrium of this intermediate subgame. Strategies map from $C_{T}^{n-1} \rightarrow C_{T}$ a typical strategy being interpreted as a contract. Consider any agent $i$ of type $t$. She will participate and choose contract $c_{t}=\left(\widehat{\gamma}_{t}, \widehat{\delta}_{t}\right)$ if and only if: ${ }^{18}$

$$
\begin{gathered}
\widehat{\gamma}_{t}+\widehat{\delta}_{t} x_{t(i)}\left(\widehat{a}_{t(i)}\left(\widehat{\delta}_{t}, \widehat{\delta}_{-i}\right), \widehat{a}_{-i}\right)-\frac{\widehat{a}_{t(i)}^{2}\left(\widehat{\delta}_{t}, \widehat{\delta}_{-i}\right)}{2} \geq 0 ; \text { and } \\
\widehat{\gamma}_{t}+\widehat{\delta}_{t} x_{t(i)}\left(\widehat{a}_{t(i)}\left(\widehat{\delta}_{t}, \widehat{\delta}_{-i}\right), \widehat{a}_{-i}\right)-\frac{\widehat{a}_{t(i)}^{2}\left(\widehat{\delta}_{t}, \widehat{\delta}_{-i}\right)}{2} \geq \\
\gamma_{t^{\prime}}+\delta_{t^{\prime}} x_{t(i)}\left(\widetilde{a}_{t(i)}\left(\delta_{t^{\prime}}, \widehat{\delta}_{-i}\right), \widetilde{a}_{-i}\right)-\frac{\widetilde{a}_{t(i)}^{2}\left(\delta_{t^{\prime}}, \widehat{\delta}_{-i}\right)}{2} ; \forall t^{\prime} \neq t
\end{gathered}
$$

Condition (1) is the participation constraint (PC) and (2) defines the set of incentive compatibility constraints (IC) for any agent $i$. Note that the expected output of the deviation payoffs includes not only another effort for agent $i$ but also for the rest of agents. The multiple-agent dimension of the model implies that when any agent deviates, the efforts of other agents do generally change. As deviations in the contract choice yield a different profile of efforts, they constrain the principal's problem (even if such deviations will not occur on the equilibrium path of the full game).

The principal's full problem: Even if the principal doesn't know who is who in the network, he knows the underlying graph of the network $g$. He can anticipate efforts, expected outputs and expected utilities for each position. Write the expected output as $x_{i}=\frac{a_{i}^{2}}{\delta_{i}}$. Then the principal's expected utility can be written as $E U_{P}=\sum_{i \in N}\left(\left(1-\widehat{\delta}_{i}\right) \widehat{x}_{i}-\right.$ $\left.\widehat{\gamma}_{i}\right)=\sum_{i \in N}\left(\frac{\left(1-\widehat{\delta}_{i}\right)}{\widehat{\delta}_{i}} \widehat{a}_{i}^{2}-\widehat{\gamma}_{i}\right)$. In matrix form: ${ }^{19}$

$$
E U_{P}=\overrightarrow{\widehat{\mathbf{d}}}^{T} \mathbf{Q} \overrightarrow{\mathbf{d}}-\overrightarrow{\widehat{\mathbf{h}}}^{T} \overrightarrow{\mathbf{1}}
$$

with $\mathbf{Q}=\left((\mathbf{I}-\beta \mathbf{D G})^{T}\right)^{-1}\left(\mathbf{D}^{-1}-\mathbf{I}\right)(\mathbf{I}-\beta \mathbf{D G})^{-1}$ and $\overrightarrow{\mathbf{h}}$ being the vector of $\gamma_{i}$ 's on the equilibrium path of the full game. Participation and incentive compatibility constraints can also be simplified by incorporating the binding Nash equilibrium conditions of the network effort subgames:

\footnotetext{
${ }^{17}$ That is, when at the first stage the principal chooses the set of contracts, he can consider effort functions as continuous in $\delta$. Once the optimal set of contracts is determined, these effort functions are not continuous. Each effort function is a collection of points, one per each particular $\delta_{t}$ offered.

${ }^{18}$ Index " $t(i)$ " denotes agent $i$ of type $t$; index " $-i$ ", all the agents but $i$. Recall that any deviation is unilateral; only agent $i$ deviates, while any other agent $j \neq i$ (including those of the same type $t$ ) does not.

${ }^{19}$ On the equilibrium path of the full game: $\sum_{i \in N}\left(1-\widehat{\delta}_{i}\right) \widehat{x}_{i}=\overrightarrow{\mathbf{a}}^{T}\left(\mathbf{D}^{-1}-\mathbf{I}\right) \overrightarrow{\widehat{\mathbf{a}}}$ where $\overrightarrow{\mathbf{a}}=$ $(\mathbf{I}-\beta \mathbf{D G})^{-1} \overrightarrow{\widehat{\mathbf{d}}}$ and $\operatorname{diag}(\mathbf{D})=\overrightarrow{\overrightarrow{\mathbf{d}}} . \quad$ It follows that $\sum_{i \in N}\left(1-\widehat{\delta}_{i}\right) \widehat{x}_{i}=\overrightarrow{\widehat{\mathbf{d}}}^{T} \mathbf{Q} \overrightarrow{\mathbf{d}}$ with $\mathbf{Q}=$ $\left((\mathbf{I}-\beta \mathbf{D G})^{T}\right)^{-1}\left(\mathbf{D}^{-1}-\mathbf{I}\right)(\mathbf{I}-\beta \mathbf{D G})^{-1}, \mathbf{Q}^{T}=\mathbf{Q}$. It is easy to see that $\mathbf{Q}$ is symmetric; for $\mathbf{D}$ is diagonal and so it is $\left(\mathbf{D}^{-1}-\mathbf{I}\right)$.
} 


$$
\begin{gathered}
\widehat{\gamma}_{t}+\frac{\widehat{a}_{t(i)}^{2}\left(\widehat{\delta}_{t}, \widehat{\delta}_{-i}\right)}{2} \geq 0, \forall t \\
\widehat{\gamma}_{t}+\frac{\widehat{a}_{t(i)}^{2}\left(\widehat{\delta}_{t}, \widehat{\delta}_{-i}\right)}{2} \geq \gamma_{t^{\prime}}+\frac{\widetilde{a}_{t(i)}^{2}\left(\delta_{t^{\prime}}, \widehat{\delta}_{-i}\right)}{2}, \forall t^{\prime} \neq t
\end{gathered}
$$

The principal's expected profit and all the constraints are now functions of the elements of the set of contract $C_{T}=\left\{\left(\gamma_{t}, \delta_{t}\right)\right\} t=1, \ldots, r$. Optimal contracts $\left\{\left(\gamma_{t}, \delta_{t}\right)\right\} t=1, \ldots, r$ maximize $E U_{P}(\mathrm{P})$ subject to the set of constraints defined by (1') and (2').

We now discuss the solution in two alternative scenarios: 1) when there is only one contract available (single contracts) and 2) when the principal offers a set of self-selecting contracts. In general, of course, the principal's expected profits rise whenever he offers a set of contracts instead of a single (pooling) contract. However, in many real life circumstances, the principal is not allowed to offer different contracts and the pooling contract is the unique alternative. We mostly rely on some simple numerical examples to show the effect of different pattern of connections and the size of the effort externality $\beta$ on optimal contracts and principal's expected profit.

\subsection{Single contracts}

Suppose that the principal offers a single contract $c=(\gamma, \delta)$ to all agents caring only about their participation. Given the contract offered, efforts are proportional to non-weighted Bonacich centralities with parameter $\delta \beta$. The principal knows in advance that agents with the minimum centrality will exert the minimum effort and expected output. He chooses the fixed term $\gamma$ such that these agents earn in expectation their reservation utility. The rest of agents will receive participation rents. Denote by $X(\delta)$ the total expected output and by $\widehat{a}_{1}(\delta)$ the effort exerted by agents with the lowest centrality.

Proposition 6 (single contract) Under A1: If the principal offers a single contract $c=$ $(\gamma, \delta)$, such that $w_{i}=\gamma+\delta y_{i} \forall i \in N$, then the optimal contract has $\widehat{\delta}$ satisfying $\widehat{\delta}=$ $1-\frac{1}{\frac{\partial X(\widehat{\delta})}{\partial \delta}}\left[X(\widehat{\delta})-n \widehat{a}_{1}(\widehat{\delta}) \frac{\partial \widehat{a}_{1}(\widehat{\delta})}{\partial \delta}\right]$ and $\widehat{\gamma}=-\frac{\widehat{a}_{1}^{2}(\widehat{\delta})}{2}$. Efforts are $\overrightarrow{\mathbf{a}}=\widehat{\delta}(\mathbf{I}-\widehat{\delta} \beta \mathbf{G})^{-1} \overrightarrow{\mathbf{1}}$. When the network is $k$ - regular, $\widehat{\gamma}(k)=-\frac{1}{2(1-2 k \beta)^{2}}$ and $\widehat{\delta}(k)=\frac{1}{1-\beta k}$ and the first best is achieved; otherwise $E \widehat{U_{P, S C}}<E U_{P}^{*}$.

Proof. In the Appendix.

Agents' expected utility depends on their position in the network. Each agent $i$ of type $t$ earns in expectation the increment in her effort-cost with respect to the agents of lowest centrality, type 1: $E U_{t(i)}=\frac{\widehat{a}_{t}^{2}(\widehat{\delta})}{2}-\frac{\widehat{a}_{1}^{2}(\widehat{\delta})}{2} \geq 0 .^{20}$

How do the structure of the network and the size of the local externality $\beta$ affect the incentive intensity $\widehat{\delta}$ and the expected utility of the principal? We will illustrate the relation between $\widehat{\delta}$ and $\beta$ for some paradigmatic networks. We measure the cost for the principal in terms of his expected utility through the ratio $\frac{E \hat{U}_{P, S C}}{E U_{P}^{*}}$ as a function of $\beta$, where $E \hat{U}_{P, S C}$

\footnotetext{
${ }^{20}$ The expected utility of an agent $i$ of type $t$ is given by: $E U_{t(i)}=\widehat{\gamma}+\widehat{\delta} \widehat{x}_{t(i)}(\widehat{\delta})-\frac{\widehat{a}_{t(i)}^{2}(\widehat{\delta})}{2}=-\frac{\widehat{a}_{1}^{2}(\widehat{\delta})}{2}+$ $\widehat{a}_{t}^{2}(\widehat{\delta})-\frac{\widehat{a}_{t}^{2}(\widehat{\delta})}{2}$. Recall that from best responses in the effort subgame, $\widehat{\delta} \widehat{x}_{t}(\widehat{\delta})=\widehat{a}_{t}^{2}(\widehat{\delta})$.
} 
$\left(E U_{P}^{*}\right)$ is the principal's expected utility under the single contract (first best). This ratio allows us to compare different networks, as first best allocations change along with the pattern of interactions. In all the networks discussed below $n=12$, and with the exception of the exclusive groups networks, all are connected. ${ }^{21}$

Given $\beta$, through a high-powered incentive $\widehat{\delta}$ the principal induces high efforts. There are two effects: 1) given the efforts of the neighbors of any agent, a higher marginal participation on her output motivates her to exert a higher effort (direct effect); and 2) since $\delta$ scales $\beta$, network externalities are stronger (indirect effect).

As in equilibrium $\widehat{\delta} \widehat{x}_{t(i)}=\widehat{a}_{t(i)}^{2}$, the principal pays in expectation to each agent two times her cost; while through the fixed term $\widehat{\gamma}$, the principal recovers from each agent an amount equivalent to the cost exerted by the agents with the lowest centrality. A high value of $\widehat{\delta}$ increases total output, leaves the principal with a lower share, but at the same time as $\widehat{a}_{1}(\widehat{\delta})$ is larger, raises his fixed profits; A low value of $\widehat{\delta}$ reduces total output and fixed profits, but augments the principal's marginal participation.
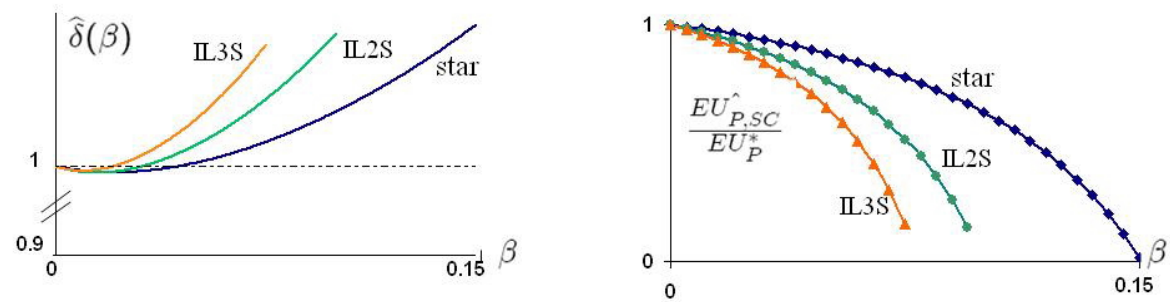

(a) - Star and interlinked stars
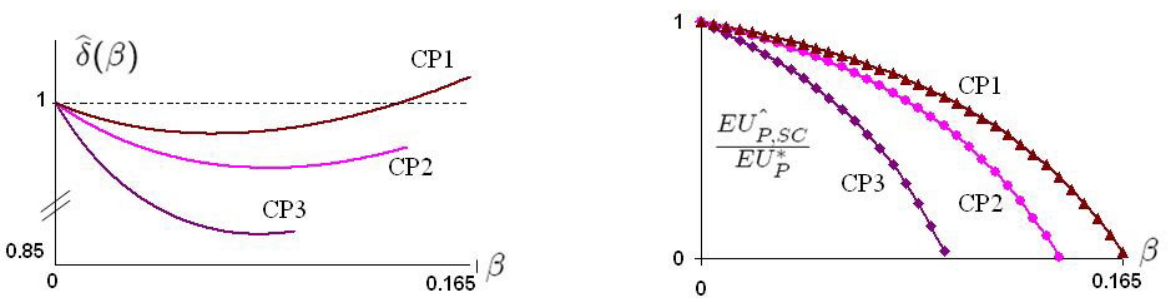

(b) - Core-periphery networks

(figure 1)

Our examples show that the incentive intensity $\widehat{\delta}$ can be $\gtreqless 1$, depending on the particular network and interaction strength $\beta$. The relation between $\widehat{\delta}$ and $\beta$ is in general nonmonotone, but smooth. With the exception of the exclusive group(s) networks (figure 2), the function $\widehat{\delta}(\beta)$ has a minimum. When peer effects are weak (small $\beta$ ), the principal finds it optimal to increase his participation on total output (decreasing $\widehat{\delta}$ ), at the cost of low fixed returns. Network externalities are not so important, and the principal will not be able to recover through the fixed terms his share on total output because agents with the lowest centrality exert too little effort. On the contrary, when $\beta$ is sufficiently high (increasing

${ }^{21}$ The graphs are given in the Appendix. 
$\widehat{\delta}(\beta))$, the principal gives a higher marginal participation to agents but obtains higher fixed profits.

Remark A more formal argument goes as follows: Consider the self-consistency condition for $\widehat{\delta}, \widehat{\delta} \lesseqgtr 1 \Longleftrightarrow \frac{X}{n} \gtreqless x_{1}+\widehat{\delta} \beta \widehat{a}_{1} \Psi_{1} \cdot{ }^{22}$ Here $\frac{X}{n}$ is the average expected output, $x_{1}$ the expected output of type 1 and $\widehat{\delta} \beta \widehat{a}_{1} \Psi_{1}$ is the marginal increase in type 1's output due to indirect effects $\left(\Psi_{1}=\sum_{j \in N_{1(i)}(g)} \frac{\partial a_{t^{\prime}(j)(\widehat{\delta})}}{\partial \delta}\right.$, see footnote 22$)$. When $\beta$ is very small, indirect effects are negligible for any reasonable $\delta$, therefore $\frac{X}{n}>x_{1}+\widehat{\delta} \beta \widehat{a}_{1} \Psi_{1}$ and $\widehat{\delta}<1$. As long as $\beta$ increases, by decreasing $\delta$ he still induces an average output higher than $x_{1}+\widehat{\delta} \beta \widehat{a}_{1} \Psi_{1}$. This continues until $\beta$ is so high that it is optimal to set $\delta$ so that the inequality is reversed. Of course this effect is more important when the fraction of agents with lowest centrality is relatively large, as in the star or in the interlinked stars (figure 1-(a)). Only the hubs obtain participation rents and they interact with all the other agents. Therefore, when $\beta$ is relative high, the principal gives a high share on outputs, so that the hubs' externalities are larger.

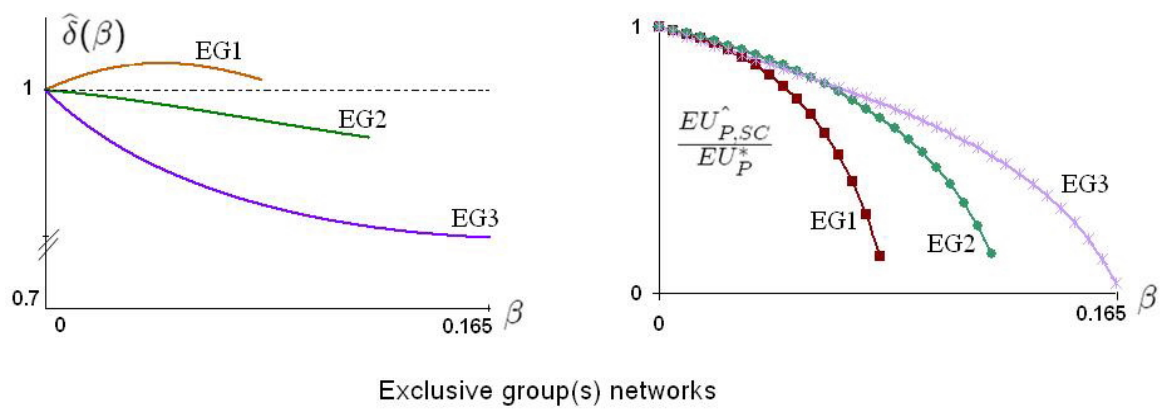

(figure 2)

A special case is the exclusive group network $E G 1$ (figure 2). There are two separate $k_{\rho}$-regular components $(\rho=1,2)$, with similar connectivity $k_{1}<k_{2}$. Here, for any given $\beta$ and $\delta$, participation rents are paid to all agents in the larger component. However, as the difference $k_{2}-k_{1}$ is small, such rents are small. For $\beta$ small, it is optimal to give agents an increasing share on their output, and the opposite when $\beta$ is large. In $E G 2$ and $E G 3$, $\widehat{\delta}$ decreases monotonically along with $\beta$.

With respect to the principal's benefits, note that in equilibrium, agents in different positions exert efforts such that their ratio of marginal cost of effort to marginal productivity

\footnotetext{
${ }^{22}$ Using best response functions,

$\widehat{a}_{1(i)}(\widehat{\delta}) \frac{\partial \widehat{a}_{1(i)}(\widehat{\delta})}{\partial \delta}=x_{1(i)}(\widehat{\delta})+\widehat{\delta} \beta \widehat{a}_{1(i)}(\widehat{\delta}) \sum_{j \in N_{1(i)}(g)} \frac{\partial a_{t^{\prime}(j)(\widehat{\delta})}}{\partial \delta} ;$ simplifying notation and as for any agent of type $1, \sum_{j \in N_{1(i)}(g)} \frac{\partial a_{t^{\prime}(j)(\widehat{\delta})}}{\partial \delta}$ takes the same value, $\widehat{a}_{1} \frac{\partial \widehat{a}_{1}}{\partial \delta}=x_{1}+\widehat{\delta} \beta \widehat{a}_{1} \Psi_{1}$ (note that $\Psi_{1}>0$ ). From the self-consistency condition for $\widehat{\delta}, \widehat{\delta} \lesseqgtr 1 \Longleftrightarrow \frac{X(\widehat{\delta})}{n} \gtreqless \widehat{a}_{1}(\widehat{\delta}) \frac{\partial \widehat{a}_{1}(\widehat{\delta})}{\partial \delta}=x_{1}+\widehat{\delta} \beta \widehat{a}_{1} \Psi_{1}$.
} 
are all equal to $\widehat{\delta}^{23}$ This constraints the possibilities for the principal to achieve the first best, as such first best ratios are increasing along with the centrality. ${ }^{24}$ From our examples we learn that the relative inefficiency (measure by $\frac{E \hat{U}_{P, S C}}{E U_{P}^{*}}$ ) increases with $\beta$ for any given network.
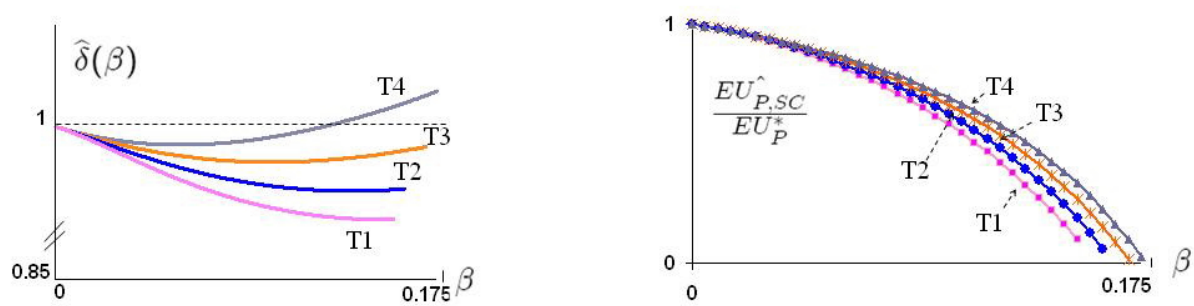

(a) - Tree networks (with 4 types)
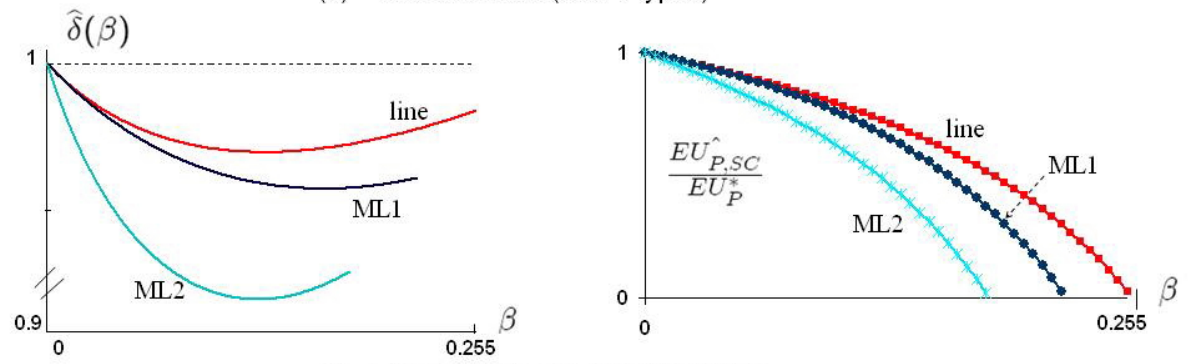

(b) - Line or chain and "modified" lines

(figure 3)

Across networks, we would like to stress some observations. First, and quite intuitively, the relative inefficiency is in general larger when the variability of Bonacich centralities is larger. ${ }^{25}$ Second, with respect to topological properties of the networks, when networks are more densely connected, the inefficiency is larger (for any $\beta$ ) than when networks are sparse. (See for example the star vs. the interlinked stars, the core-periphery networks or the lines and "modified lines"). For networks with the same density and the same number of types, the principal loses relatively more when the variance of the connectivity is higher. We show this through the case of the trees with four types (figure $3-(\mathrm{a})) .{ }^{26}$ The ratio $\frac{E \hat{U_{P, S C}}}{E U_{P}^{*}}$ is also

\footnotetext{
${ }^{23}$ Marginal cost is $M C_{t}=\widehat{a}_{t(i)}$, marginal productivity $M P_{t}=1+\beta \sum_{j \in N_{i}(g)} \widehat{a}_{t^{\prime \prime}(j)}$; then $\widehat{\delta}=\frac{M C_{t}}{M P_{t}}=$ $\frac{M C_{t^{\prime}}}{M P_{t^{\prime}}} \forall t \neq t^{\prime}$.

${ }^{24}$ Indeed, it is quite easy to see that such ratios are increasing along with the type in the first best case. From contracts offered under moral hazard (Prop. 2) that allow the principal to achieve the first best, and using the same notation as in the proof of Prop. 4:

$\frac{M C_{t}}{M P_{t}}=\delta_{t(i)}=1+\frac{\Phi_{t}^{*}-1}{\Phi_{t}^{*}}=\frac{2 \Phi_{t}^{*}-1}{\Phi_{t}^{*}}$

$\frac{M C_{t}}{M P_{t}}-\frac{M C_{t^{\prime}}}{M P_{t^{\prime}}}=\frac{\Phi_{t}^{*}-\Phi_{t^{\prime}}^{*}}{\Phi_{t}^{*} \Phi_{t^{\prime}}^{*}}>0 \Longleftrightarrow t>t^{\prime}$.

${ }^{25}$ The latter can be measured as the standard deviation of first best efforts, for a given $\beta$ in the range s.t. all networks can be compared.

${ }^{26}$ The structure of each tree is similar. There are two agents interconnected, and each of them linked to other (end) nodes, $n_{1}$ and $n_{2}$ respectively, with $n_{1}<n_{2}$ and $n_{1}+n_{2}=n-2$. In all of them,
} 
negatively correlated to the average clustering (see the core-periphery networks in figure $1-(b))$.

\subsection{Menu of contracts}

In this subsection, we assume that the principal offers a menu of contracts $C_{T}=\left\{\left(\gamma_{t}, \delta_{t}\right)\right\}, t=$ $1, \ldots, r$, where contract $c_{t}=\left(\gamma_{t}, \delta_{t}\right)$ is directed towards agents of Bonacich type $t$. The optimal set $C_{T}$ solves the complete program defined by $(\mathrm{P})$ and the set of constraints (1), (2). Typically in these kind of models, the main difficulty resides in reducing the number of constraints to a more tractable set. We have already relaxed the restrictions on the effort functions, since we know that Nash equilibria at that stage are unique and interior for each $\overrightarrow{\mathbf{d}}$. With respect to the rest of constraints, we also know that the cost of the principal is minimized if informational rents are minimized and that this occurs whenever any agent of type $t$ is exactly indifferent between contract $c_{t}$ and $c_{t-1}$. Now the principal has $r \times 2$ instruments to induce such behavior.

First, the principal will find it optimal to induce on-the-equilibrium-path efforts that preserve the order of the non-weighted Bonacich centralities. In other words, agents' efforts will be increasing in type. A sufficient condition is that incentive intensities $\delta_{t}$ are weakly increasing along with types. It follows that if $\delta_{t} \geq \delta_{t^{\prime}} \Longleftrightarrow t \geq t^{\prime}$, necessarily, $\gamma_{t} \leq \gamma_{t^{\prime}}$, or else agents of (lower) type $t^{\prime}$ would "imitate" (higher) type $t$. Second, through the marginal incentives the principal is able to induce (on- and off-the-equilibrium-path) efforts of agents in different positions (types) such that agents reveal their types at the minimum cost for the principal. As the effort of any agent can be interpreted as an increasing and strictly convex function of her incentive intensity (see Prop. 5), the principal can affect effort functions by manipulating marginal participations. In Prop. 7 we assume that these relations are induced, and show that the set of relevant constraints are the local downward incentive compatibility and the participation constraint of the lowest type $(t=1)$. Contracts with these features are incentive compatible and individual rational (all agents participate). Agents of high centrality reveal their type if they are paid informational rents, and the minimum rent is is such that any type $t$ is exactly indifferent between contract $c_{t}$ and $c_{t-1}$. Prop. 7 state the sufficient conditions.

Proposition 7 (menu of contracts) Assume A1. If the principal offers a set of contracts $C_{T}=\left\{\left(\widehat{\gamma}_{t}, \widehat{\delta}_{t}\right)\right\}, t=1, \ldots, r \quad\left(w_{t(i)}\left(y_{i}\right)=\widehat{\gamma}_{t}+\widehat{\delta}_{t} y_{i}\right)$ with $\widehat{\delta}_{t} \geq \widehat{\delta}_{t-1}, \widehat{\gamma}_{t} \leq \widehat{\gamma}_{t-1} \forall t$ such that $a_{t(i)}\left(\delta_{\tau}, \widehat{\delta}_{-i}\right) \geq a_{t^{\prime}(j)}\left(\delta_{\tau}, \widehat{\delta}_{-j}\right)$ and $a_{t(i)}\left(\delta_{\tau}, \widehat{\delta}_{-i}\right)-a_{t(i)}\left(\delta_{\tau^{\prime}}, \widehat{\delta}_{-i}\right) \geq a_{t^{\prime}(j)}\left(\delta_{\tau}, \widehat{\delta}_{-j}\right)-$ $a_{t^{\prime}(j)}\left(\delta_{\tau^{\prime}}, \widehat{\delta}_{-j}\right) \forall t \geq t^{\prime}, \forall \delta_{\tau}, \delta_{\tau^{\prime}} \in C_{T}: \delta_{\tau} \geq \delta_{\tau^{\prime}}$, then the set of relevant constraints are the local downward (IC)-conditions and the participation constraint of type 1. For any (non

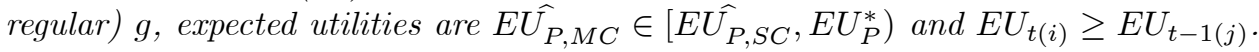

Proof. In the Appendix.

$\bar{k}=\frac{2\left(n_{1}+n_{2}+1\right)}{n}=2 \frac{n-1}{n}$. The second moment is $\sum_{i} \frac{k_{i}^{2}}{n}=(n-1)-2 n_{1}\left(1-\frac{n_{1}+2}{n}\right)$. Then $\operatorname{Var}(k)_{T 1}>$ $\operatorname{Var}(k)_{T 2}>\operatorname{Var}(k)_{T 3}>\operatorname{Var}(k)_{T 4}$. 

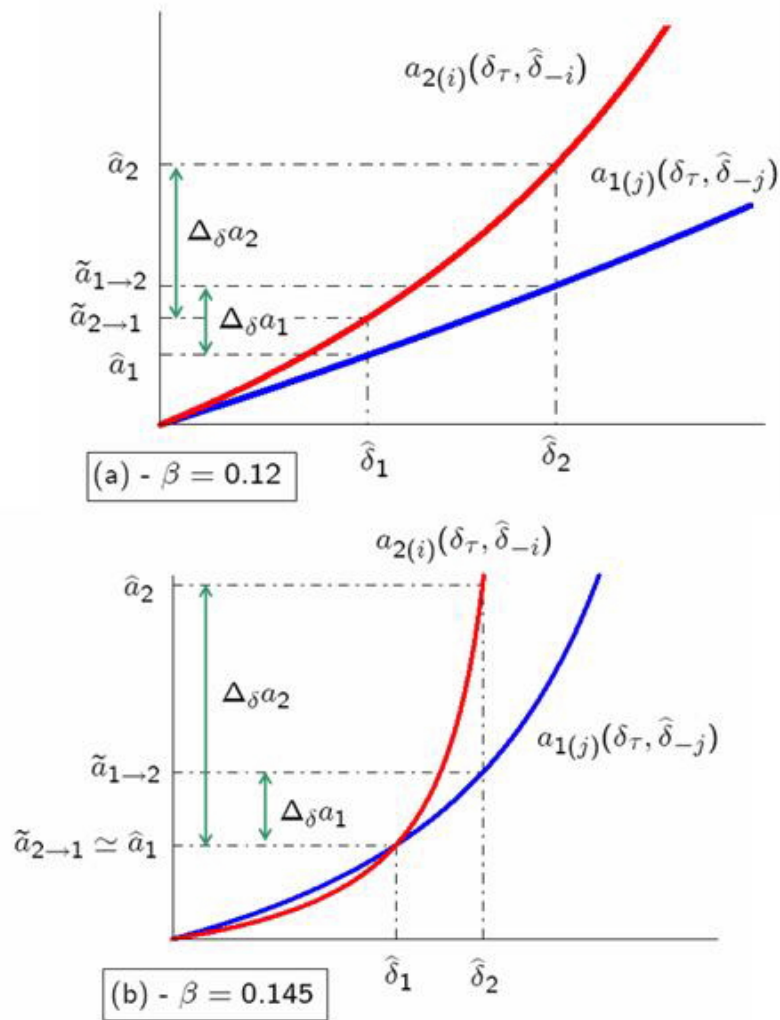

(figure 4)

It should be clear that $a_{t(i)}\left(\delta_{\tau}, \widehat{\delta}_{-i}\right)$ is the equilibrium effort of agent $i$ of type $t$ in the subgame when all other agents choose the "right" contract and agent $i$ chooses contract $\delta_{\tau}$. If $\delta_{\tau}=\widehat{\delta}_{t}$, then $a_{t(i)}\left(\delta_{\tau}, \widehat{\delta}_{-i}\right)=a_{t(i)}\left(\widehat{\delta}_{t}, \widehat{\delta}_{-i}\right)$ is $i$ 's effort on the equilibrium path of the full game. On the other hand, $a_{t^{\prime}(j)}\left(\delta_{\tau}, \widehat{\delta}_{-j}\right)$ is the effort of any agent $j$ of a lower type than $i$, in the subgame where all the other agents are choosing the right contract, while $j$ chooses $\delta_{\tau}$. We illustrate what Prop. 7 implies in figure 4. The figure depicts the case of a star of $n=12$ agents, for $\beta=0.12$ (panel (a)) and $\beta=0.145$ (panel (b)). The effort of type 2, the hub, is evaluated for all spokes choosing $\widehat{\delta}_{1}$, while effort of type 1 , any of the spokes, is evaluated at all the $n-2$ spokes choosing $\widehat{\delta}_{1}$ and the hub, $\widehat{\delta}_{2}$.

The principal sets fixed terms $\widehat{\gamma}_{t}$ in terms of the incentive intensities so that total cost is minimized. That is, $\widehat{\gamma}_{1}=-\frac{\widehat{a}_{1(i)}^{2}\left(\widehat{\delta}_{1}, \widehat{\delta}_{-i}\right)}{2}$ and $\widehat{\gamma}_{t}=\widehat{\gamma}_{t-1}-\left(\frac{\widehat{a}_{t(i)}^{2}\left(\widehat{\delta}_{t}, \widehat{\delta}_{-i}\right)}{2}-\frac{\widetilde{a}_{t(i)}^{2}\left(\delta_{t-1}, \widehat{\delta}_{-i}\right)}{2}\right)$.

We rely on the same numerical examples as in Section 3.1 to show more details of the incentives' schemes for different networks. Proposition 7 doesn't account for one important result: Full separation among different types is not always optimal. Depending on the particular network and the degree of interaction parametrized by $\beta$, the principal sometimes obtains higher profits if he offers a set of contract $C_{T}$ with (almost) partial bunching of types. $^{27}$ Formally this occurs whenever some constraint $\widehat{\delta}_{t} \geq \widehat{\delta}_{t-1}$ almost binds. In these

\footnotetext{
${ }^{27}$ In partial bunching contracts, two marginal incentives $\delta_{t}, \delta_{t^{\prime}}$ are equal up to six (6) decimal places.
} 
cases, the principal offers less contracts than there are types as some contracts are intended for agents of different types.

We depict optimal marginal incentives, $\widehat{\delta}_{t}(\beta)$ and expected utilities of agents (since $\underline{U}=0, E U_{t}$ is equal to the informational rent type $t$ receives). Again we measure the principal's losses with respect to the first best with the ratio $\frac{E U_{P, M C}^{*}}{E U_{P}^{*}}$ and we show the relation between the effort induced by self-selecting contracts and the first best effort for each type $t, \frac{\widehat{a}_{t}}{a_{t}^{*}}$.
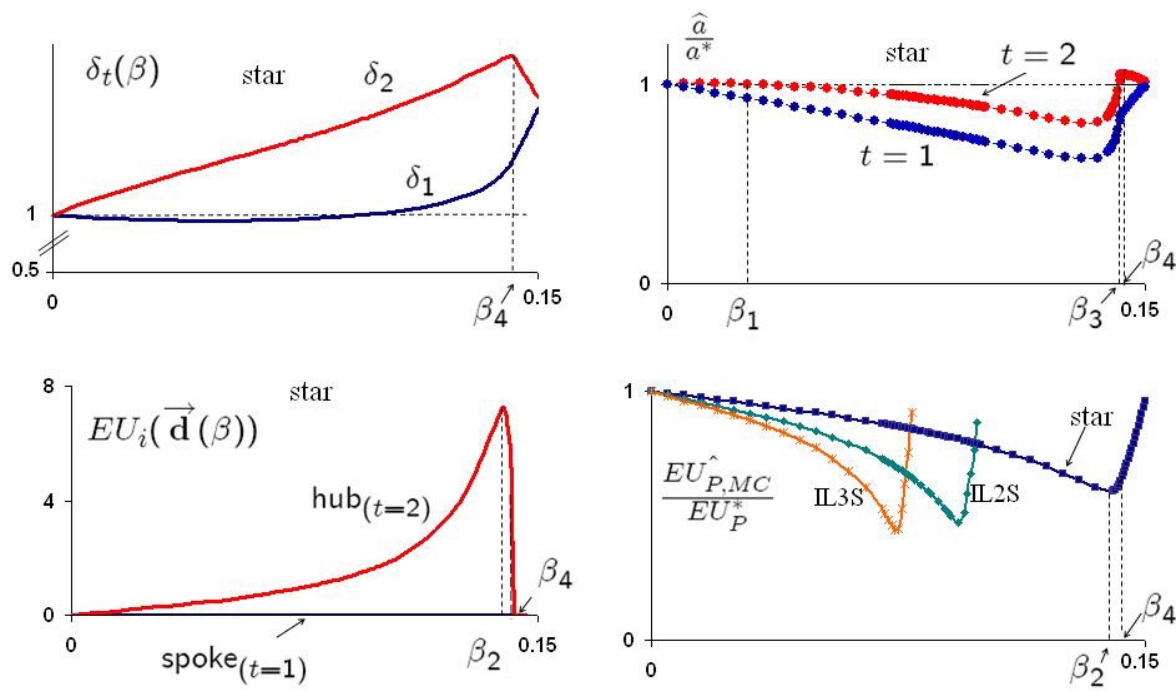

Star and interlinked stars

(figure 5)

Connected Networks Consider the connected networks with 2 types, star and interlinked stars and core-periphery networks. Single contracts are never optimal (unless $\beta=0$ ) and thus the principal always offers two contracts. As long as $\beta$ increases, the principal offers an increasing share to agents with higher centrality. This continues until $\beta$ is sufficiently high where both marginal incentives start to converge $\left(\widehat{\delta}_{2}\right.$ decreases and $\widehat{\delta}_{1}$ increases $)$. The difference $\widehat{\delta}_{2}-\widehat{\delta}_{1}$ is thus maximized for some intermediate $\beta$. The intuition isas follows. When $\beta$ is very small peer effects are negligible, and efforts are $\widehat{a}_{t} \simeq \widehat{\delta}_{t}$. Perfect revelation is obtained at very small costs. On the other extreme, when interaction is intense, the principal optimally sets the different incentive intensities such that when the high type (the hubs in the stars or the centers in the core-periphery networks) imitates the low type, her induced effort is "almost" equal to the effort of the low type on the equilibrium path.

However, while the principal doesn't pay informational rents, the first best is not achieved. $^{28}$ See for example the ratio of principal's utilities of the star (figure 5). There

\footnotetext{
That's why we add the term "almost."

${ }^{28}$ Note that in all the networks where there are two types, the principal can choose different contracts such that both types are left on their reservation utility. This is not optimal when $\beta$ is low, because as necessarily $\widetilde{a}_{2}\left(\delta_{1}, \widehat{\delta}_{-i}\right) \simeq \widehat{a}_{1}\left(\widehat{\delta}_{1}, \widehat{\delta}_{-j}\right)$, the principal will have to set $\delta_{1}$ at a very low value, and both types'
} 
are several "critical" values of $\beta$ ranked as follows $\beta_{1}<\beta_{2}<\beta_{3}<\beta_{4}$. $\beta_{4}$ is the value above which the principal doesn't pay informational rents to the hub (and corresponds roughly to the maximum incentive intensity offered to these agents). Still the ratio of expected utilities is lower than 1 until $\beta$ is at its upper bound. The reason is that even if the principal doesn't pay informational rents, he is distorting efforts. In the interval $\left(\beta_{1}, \beta_{3}\right)$, all agents are induced to exert lower efforts than in the first best. At the extremes, we observe that the hubs ' efforts are oversupplied. At last, for $\beta_{2}$ the inefficiency is maximal (minimum $\left.\frac{E U_{P, M C}^{\wedge}}{E U_{P}^{*}}\right)$. For the interlinked stars, the interval $\left(\beta_{1}, \beta_{3}\right)$ is larger. In the case of core-periphery networks, we observe that center nodes exert higher efforts than in the first best only for $\beta$ close to $\bar{\beta}$ (see in figure 6 the ratio of efforts for $C P 1$ and $C P 3$ ).
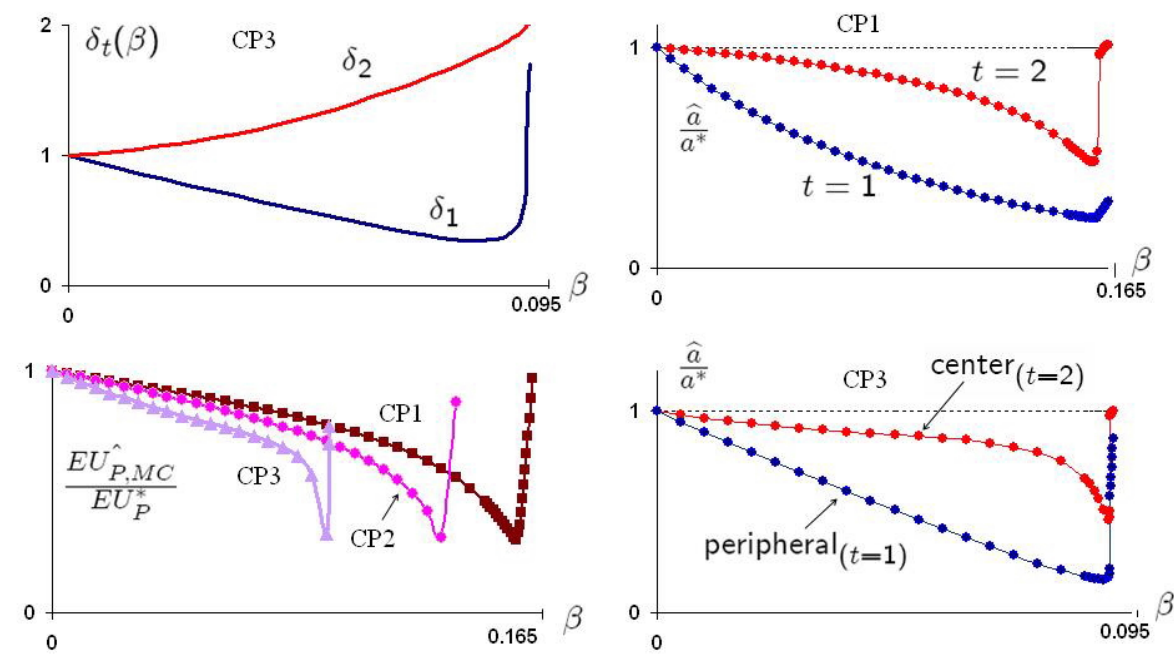

Core-periphery networks

(figure 6)

Exclusive Group Networks In the exclusive groups network $E G 1$ there are also two types (figure 7 ). This is the only example in which the single contract is optimal for a range of $\beta \in\left[0, \beta_{0}\right), \beta_{0}<\bar{\beta}$. The reason is that when $\beta$ is relatively small, agents in different groups have similar centralities. If the principal offers a single contract, agents in the larger group receive participation rents. It turns out that choosing the same marginal participation $\delta_{\{1,2\}}$ for both types these rents are less than the informational rents that the same agents would receive. ${ }^{29}$ Another characteristic of exclusive groups is that when groups are complete subgraphs (as they are in our examples), the ratio $\frac{\widehat{a}_{t}}{\widehat{a}_{t}^{*}}$ is approximately proportional to $\widehat{\delta}_{t}$. This is simply because when all $k_{\rho}$ agents within a group are of the

efforts will be exaggeratedly downward distorted.

${ }^{29}$ Recall that under the single contracts, any agent of type 2 receives $E U_{2(i)}=\frac{\widehat{a}_{2}^{2}(\widehat{\delta})}{2}-\frac{\widehat{a}_{1}^{2}(\widehat{\delta})}{2}$. With a menu of contracts with $\widehat{\delta}_{2}>\widehat{\delta}_{1}, E U_{2(i)}=\frac{\widetilde{a}_{2}^{2}\left(\delta_{1}, \widehat{\delta}_{-i}\right)}{2}-\frac{\widehat{a}_{1}^{2}\left(\widehat{\delta}_{1}, \widehat{\delta}_{-j}\right)}{2}$. When $\beta$ is relatively low, and since the components are complete, when any agent of type 2 imitates type 1 , her effort $\widetilde{a}_{2}^{2}\left(\delta_{1}, \widehat{\delta}_{-i}\right) \simeq \widehat{a}_{2}^{2}\left(\widehat{\delta}_{2}, \widehat{\delta}_{-i}\right)$, unless $\widehat{\delta}_{1}$ is very low. But then, if $\widehat{\delta}_{1}$ is very low, the surplus of low types are low, and this affects negatively the principal's profits. 
same type, the effort of any of them is $\widehat{a}_{t}=\frac{\widehat{\delta}_{t}}{1-\widehat{\delta}_{t} \beta k_{\rho}}$, while in the first best, $a_{t}^{*}=\frac{1}{1-2 \beta k_{\rho}}$.

In all the networks where there are more than two types, we observe that there will be bunching at the optimum. That is, the principal offers some contracts which are intended for more than one type. For a given network, which types will be pooled depend on the strength of interaction $\beta$. For example, in $E G 2$ (figure 7), there are three complete components of different connectivity. For any $\beta$, the principal offers two contracts, one for low types, with incentive intensity $\delta_{\{1,2\}}$, the other for the agents in the largest component $(t=3)$. In $E G 3$, there are 4 positions, including one isolated agent. Unless $\beta$ is large, the principal offers three contracts, pooling agents of types 2 and 3 . When $\beta$ is large, he offers two contracts, only discriminating agents in the largest component.
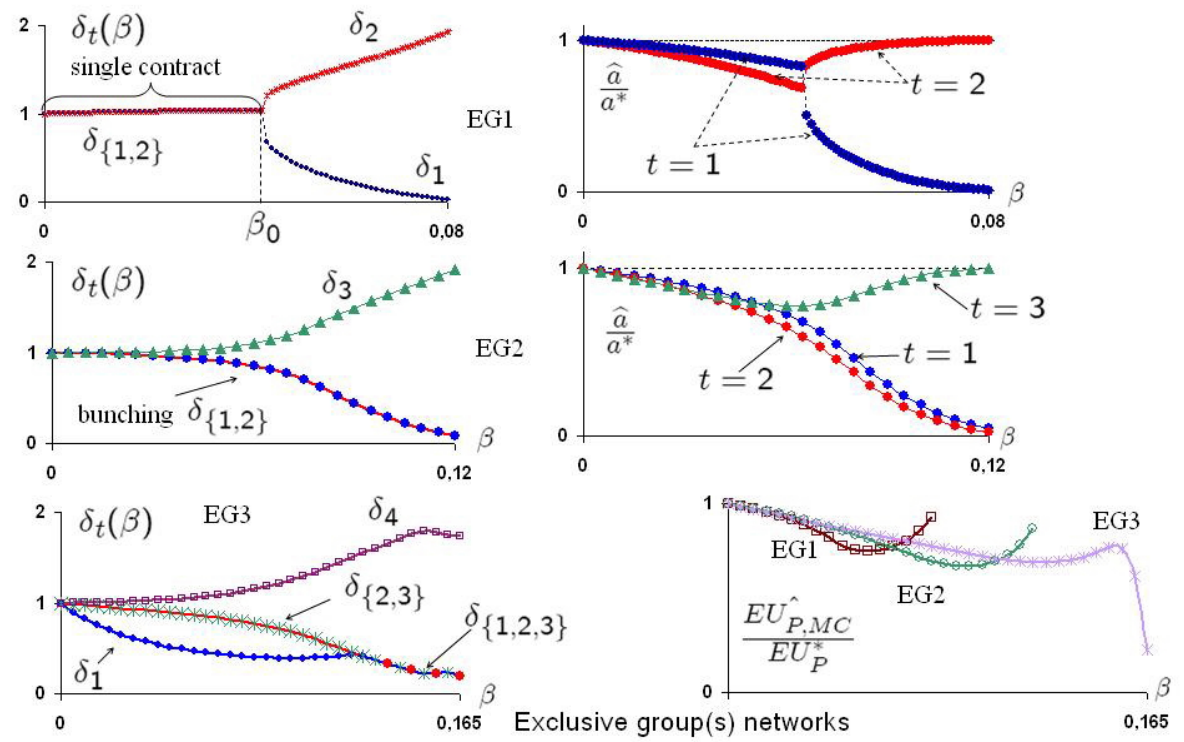

(figure 7)

Tree Networks In general, the qualitative relation between the network structure, peer effect's parameter $\beta$ and contracts becomes more complicated as the number of types increases. See for instance the tree networks with 4 types (figure 8; for $T 1$, not shown, the contracts are similar to $T 2$ ). As we mentioned before, these networks display the same average connectivity but different dispersion. In the case of $T 3$, the relation between the incentive intensities and $\beta$ is not always "smooth." For $\beta$ low, two contracts are offered, one for high types, the other for low types. As $\beta$ increases, the principal offers a different contract for the highest type. But then, there is a higher value of $\beta$ above which the principal pools intermediate types. This last transition implies a dramatic change in marginal incentives for all agents. For some interaction patterns, the degree of bunching decreases with $\beta$. Consider, for example, the line (figure 10). As long as $\beta$ increases, the principal offers more contracts, separating progressively lower types, i.e. agents at the end of the line $(t=1)$, agents connected to them $(t=2)$, and so on. In the case of $M L 2$, the behavior is similar, and it is the unique case with more than two types in which (for $\beta$ high) full separation is 
optimal. Full separation can be also optimal for intermediate values of $\beta$, as it is shown in figure 9 , for a tree with 4 types, but $n=8$.

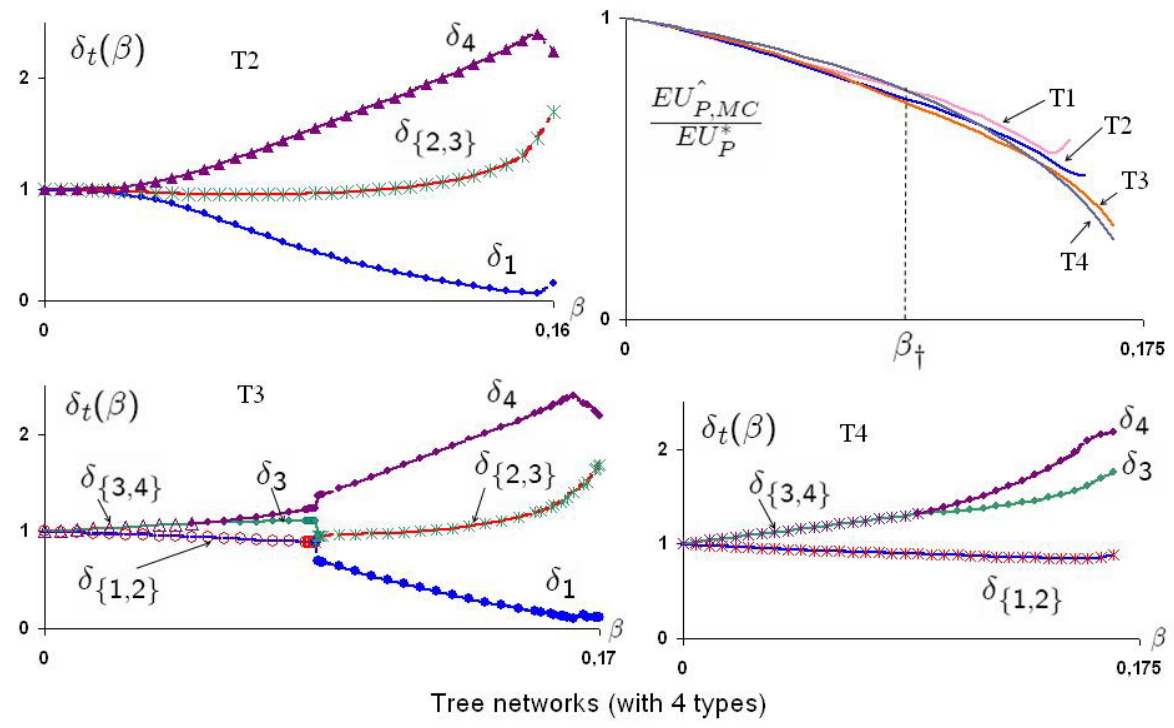

(figure 8)

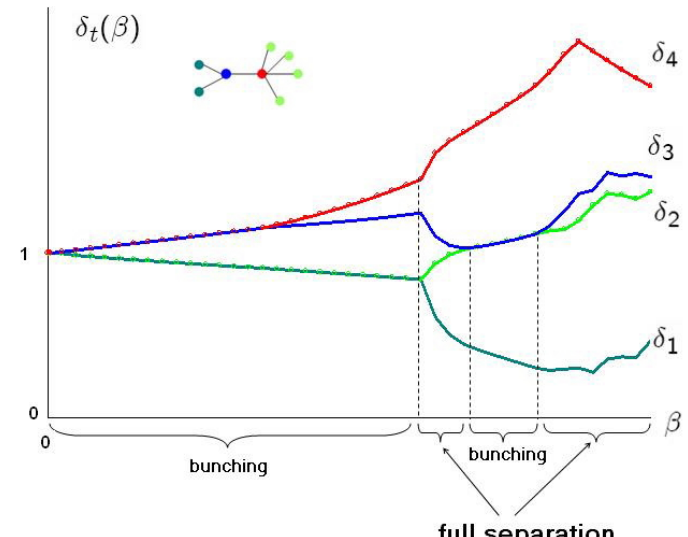

Full separation for intermediate values of $\beta$

(figure 9) 

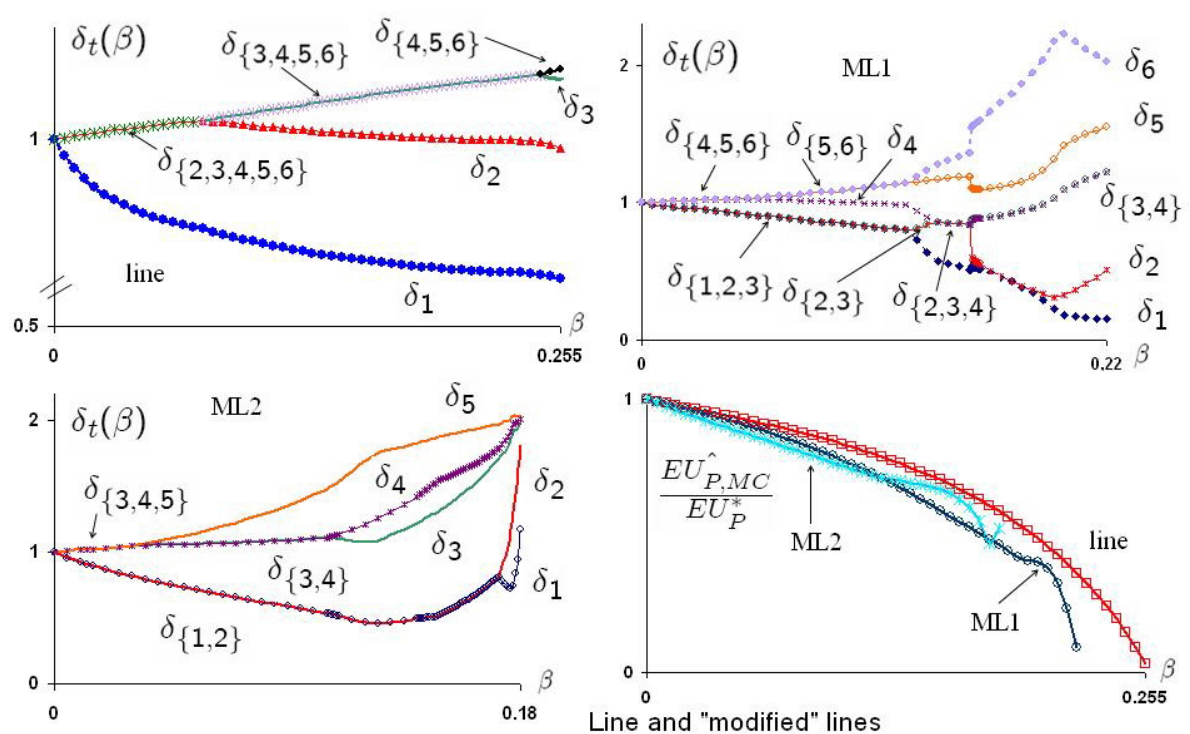

(figure 10)

The principal's profits The principal improves his profits compared to single contracts. Given $g$, the relation between $\frac{E U_{P, M C}^{*}}{E U_{P}^{*}}$ and $\beta$ can be non-monotonous, specially when there are two types. Across networks, the relation between some topological properties of $g, \beta$ and the ratio $\frac{E U_{P, M C}^{*}}{E U_{P}^{*}}$ may change. As an example consider again the tree networks (figure 8 ). Recall that when the principal offers a single contract, for all $\beta$, he loses more when the variance of connectivity is high. With the menu of contracts, this relation depends on $\beta$. Compare the networks $T 1$ and $T 4$ (highest and lowest variance, respectively). In practice, the first network is almost a star except for the agent connected to one of the "spokes." On the contrary, in $T 4$ there are two hubs, one more connected than the other. In the interval $\left(0, \beta_{\dagger}\right)$, the principal obtains the maximum ratio for $T 4$, above $\beta_{\dagger}$, for $T 1{ }^{30}$

In the last figure (figure 11) we show for some of the networks the ratio $\frac{E U_{P, S C}^{\wedge}}{E U_{P, M C}^{*}}$, as a measure of how costly is to offer a pooling contract instead of the set of contracts. A small ratio implies a high cost in terms of utility. When peer effects are important, for some networks the single contract may be really counterproductive, as for the star or the core-periphery network. ${ }^{31}$

\footnotetext{
${ }^{30}$ The relation among the ratios for the other two networks also changes. Indeed, for $\beta$ low the order is the same as with single contracts; then progressively, we observe how the order changes, until it is completely reversed for high $\beta$.

${ }^{31} \mathrm{This}$ result is in some sense related to Winter (2004). In a principal-multiple agents model with identical agents, Winter finds that optimal incentive mechanisms may require that agents be rewarded differentially even if they are identical and are induced to act the same. Discrimination is optimal if and only if the technology has increasing returns to scale. In our model the technology has increasing returns to scale,
} 


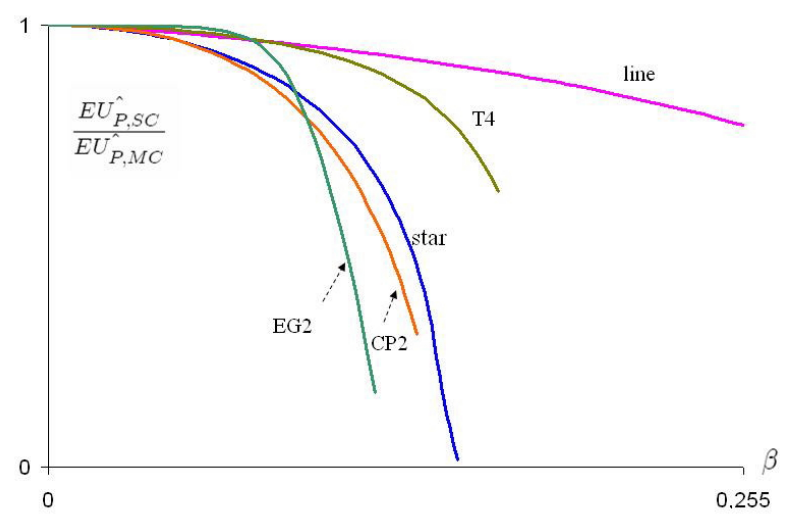

(figure 11)

In synthesis, when the principal offers a set of contracts, highly central agents are given high-powered incentives (high $\delta_{t}$ ), while low types' marginal incentives are low-powered. If there are more than two types, the principal may find it optimal to offer the same contract(s) to agents in different positions (bunching). The structure of the network together with the strength of peer effects determine which types will be optimally pooled and shape the level of the incentive intensity offered to each type. Even if full separation is not always optimal, some degree of discrimination may be preferable. When such discrimination is possible (i.e. if the principal can offer different contracts) and peer effects are strong, for some simple networks as the star, the principal is able to reduce considerably his losses with respect to the full information setting. Furthermore, always if peer effects are important, some topological properties of the networks may be valuable for the principal, as for instance, a high variability in the agents' connectivity. Although the principal obtains higher profits than with a single contract, there may be additional administrative costs - not considered here - associated to the menu of contracts. If the size of effort externality is relatively low, for some structures these additional costs can offset higher profits. However, for some networks, in particular those with high variability in the connections or with high clustering, probably it would be still convenient to offer different contracts.

\section{Conclusions}

We have analyzed the effect of informal networks on a static principal-multiple agents model. Agents' interaction is shaped by the given network of informal contacts, where links are interpreted as mutual advise or peer effect. Thus individual outputs depend positively on own effort and nearest neighbors' efforts. The principal then should compensate agents according to their position in the network. When efforts are not observable and the principal has imperfect information about the informal network, he can induce agents to exert some desired level of efforts through incentives. In particular, we assume wages that are linear in individual outputs. The structure of the network together with the size of effort externality impinge on the structure of such incentives. Under risk neutrality and absence of limited

but agents are not induced to choose the same effort. However, if we allow only for two levels of efforts, high and low, and if the principal wants to induce all agents to choose the high effort, we would have still increasing returns to scale and offering different wages would be better than a common wage. 
liability constraints, the principal can obtain the highest profits (first best) if either he observes efforts or knows the position of each agent in the social network. When both problems, moral hazard and adverse selection, are present, the principal's best alternative is to offer a self-selecting menu of contracts. At the optimum, however, there may be less than perfect revelation, as the principal may pool some types into the same contract. The degree of bunching depends on the network and for each given network, on the strength of peer effects.

In our model, agents know exactly the whole network. The principal knows only the underlying graph, thus ignores the exact position of each agent. Of course, other informational settings can be more realistic; in this sense, this work is a first step. When informal networks are large and/or relationships are volatile, agents would have less than perfect information about the network. For example, if agents only know to whom they are connected with, the effort subgame would be interpreted as a Bayesian game where in practice, each agent estimates her neighbors' externality as $k_{i}(g)$ times the expected effort of a typical neighboring agent. ${ }^{32}$ Consistently with this information, the principal would know only the distribution of connectivity. If so, the problem becomes approximately isomorphic to a principal-one agent model. This is not necessarily a disadvantage, inasmuch as on the other hand, it would be possible to work with more complex networks than the ones we presented here, and to infer more general conclusions about different topologies.

${ }^{32}$ See also Galeotti and Vega-Redondo (2005) and Galeotti et al (2006). 


\section{References}

[1] Andolfatto, D. and E. Nosal (1997), "Optimal team contracts," Canadian Journal of Economics 30(2), 385-396.

[2] Aoki, M. (1994), "The contingent governance of teams: analysis of institutional complementary," International Economic Review 35(3), 657-676.

[3] Azoulay, P. and J. G. Zivin (2005), "Peer effects in the workplace: Evidence from professional transitions for the superstars of medicine," mimeo, Columbia University and NBER.

[4] Ballester, C., A. Calvó-Armengol and Y. Zenou (2006), "Who's who in networks. Wanted: the key player," Econometrica 74 (5), 1403-1417.

[5] Ballester, C. and A. Calvó-Armengol (2006), "Interaction patterns with hidden complementaries," mimeo.

[6] Baker, G., M. Jensen and K. Murphy (1988), "Compensation and incentives: practice vs. theory," Journal of Finance 43(3), 593-616.

[7] Bandiera, O., I. Barankay and I. Rasul (2005), "Social preferences and the response to incentives: Evidence from personnel data," Quarterly Journal of Economics (Aug.), 917-962.

[8] Bandiera, O., I. Barankay and I. Rasul (2007), "Social incentives in the workplace," mimeo, London School of Economics, University of Warwick and University College London.

[9] Bolton, P. and M. Dewatripont (2005), Contract Theory, The MIT Press.

[10] Bonacich, P. (1987), "Power and centrality: A family of measures," American Journal of Sociology 92, 1170-1182.

[11] Bramoullé, Y. and R. Kranton (2007), "Public goods in networks," Journal of Economic Theory 135 (1), 478-494.

[12] Brouwer, A. E. and W. H. Haemers (2003), "Spectra of graphs," extended lecture notes, Dept. of mathematics \& computer science, Technische Universiteit Eindhoven and Dept. of econometrics \& operations research, Tillburg University (www.cwi.nl/ aeb/math/ipm.pdf).

[13] Cabrales, A., A. Calvó-Armengol and Y. Zenou (2007), "Social interactions and spillovers: incentives, segregation and topology," mimeo, Universidad Carlos III, Universitat Autònoma de Barcelona and University of Stockholm.

[14] Calvó-Armengol, A. and J. De Martí (2007), "Communication networks: knowledge and decisions," AEA Papers and Proceedings 97 (2), 86-91.

[15] Che, Y.-K. and S.-W. Yoo (2001), "Optimal incentives for teams," American Economic Review 91(3), 525-541. 
[16] Cremer, J. (1980), "A partial theory of the optimal organization of a bureaucracy," Bell Journal of Economics 11(2), 683-693.

[17] Cross, R., L. Prusak and A. Parker (2002), "Where work happens: The care and feeding of informal networks in organizations," IKO working paper.

[18] Cross, R., S. P. Borgatti and A. Parker (2002), "Making invisible work visible: Using social network analysis to support human networks," California Management Review $44(2), 25-46$.

[19] Debreu, G. and I. N. Herstein (1953) "Nonnegative square matrices," Econometrica $21(4)$.

[20] DiMaggio, P. (2001), The Twenty-first century firm, Princeton: Princeton University Press.

[21] Drago, R. and G. Garvey (1998), "Incentives for helping on the job: theory and evidence," Journal of Labor Economics 16(1), 1-25.

[22] Falk, A. and A. Ichino (2006), "Clean evidence on peer effects," Journal of Labor Economics 24 (1), 39-57.

[23] Faynzilberg, P. and P. Kumar (2000), "On the generalized principal-agent problem: Decomposition and existence results," Review of Economic Design 5, 23-58.

[24] Feldman, M., J. Chuang, I. Stoica and S. Shenker (2005), "Hidden-action in multi-hop routing," ACM Conference on Electronic Commerce (EC'05).

[25] Galeotti, A. and F. Vega-Redondo (2005), "Strategic analysis in complex networks with local externalities," California Institute of Technology, Social Science Working Paper 1224.

[26] Galeotti, A., S. Goyal, M. Jackson, F. Vega-Redondo and L. Yariv (2006), "Network Games," ECO Working Papers.

[27] Ghatak, M. M. and T. Sjöström (2001), "Occupational choice and dynamic incentives," Review of Economic Studies 68(4), 781-810.

[28] Gibbons, R. (1998), "Incentives in organizations," Journal of Economic Perspectives 12(4), 115-132.

[29] Gould, E. D. and E. Winter (2006), "Technological peer effects between workers: Evidence from professional baseball," mimeo, The Hebrew University.

[30] Grossman, S. and O. D. Hart (1983), "An analysis of the principal-agent problem," Econometrica 51(1), 7-46.

[31] Groves, T. (1973), "Incentive in teams," Econometrica 41(4), 617-631.

[32] Guesnerie, R. and J.-J. Laffont (1984), "A complete solution to a class of principalagent problems with an application to the control of a self-managed firm," Journal of Public Economics 25, 329-369. 
[33] Gupta, S. and R. Romano (1998), "Monitoring the principal with multiple agents," Rand Journal of Economics 29(2), 427-442.

[34] Holmstrom, B. (1979), "Moral hazard and observability," Bell Journal of Economics 10(1), 74-91.

[35] Holmstrom, B. (1982), "Moral hazard in teams," Bell Journal of Economics 13(2), 324-340.

[36] Ichino, A. and G. Maggi (2000), "Work environment and individual background: explaining regional shirking differentials in a large italian firm," Quarterly Journal of Economics 115, 1057-1090.

[37] Ishiguro, S. and H. Itoh (2001), "Moral hazard and renegotiation with multiple agents," Review of Economic Studies 68(1), 1-20.

[38] Itoh, H. (1991), "Incentives to help in multi-agent situations," Econometrica 59(3), 611-636.

[39] Jullien, B., B. Salanié and F. Salanié (2007), "Screening risk-averse agents under moral hazard: single crossing and the CARA case," Economic Theory 30, 151-169.

[40] Kandel, E. and E. P. Lazear (1992), "Peer pressure and partnerships," Journal of Political Economy 100 (4), 801-817.

[41] Kleinberg, J. and P. Raghavan (2005), "Query Incentive Networks," mimeo Cornell University.

[42] Krackhardt, D. and J. R. Hanson (1993), "Informal networks: the company behind the chart," Harvard Business Review 71, 104-111.

[43] Laffont, J. J. and J. Tirole (1986), "Using cost observation to regulate firms," Journal of Political Economy 94, 614-641.

[44] Levitt, S. (1995), "Optimal incentive schemes when only the agents' 'best' output matters to the principal," Rand Journal of Economics 26(4), 744-760.

[45] Lockwood, B. (2000), "Production externalities and two-way distortion in principalmulti-agent problems," Journal of Economic Theory 92, 144-166.

[46] McAfee, R. P. and J. McMillan (1987), "Competition for Agency contracts," RAND Journal of Economics, 18, 296-307.

[47] McAfee, R. P. and J. McMillan (1991), "Optimal contracts for teams," International Economic Review 32 (3), 561-577.

[48] Malcomson, J. M. and F. Spinnewyn (1988), "The multiperiod principal-agent problem," Review of Economic Studies 55, 391-408.

[49] Mas, A. and E. Moretti (2006), "Peers at work," IZA Discussion Paper 2292.

[50] Mirrlees, J. A. (1971), "An exploration in the theory of optimal income taxation," Review of Economic Studies 38, 175-208. 
[51] Mirrlees, J. A. (1976), "The optimal structure of incentives and authority within an organization," The Bell Journal of Economics 7(1), 105-131.

[52] Mirrlees, J. A. (1999), "The theory of moral hazard and unobservable behaviour: Part I," Review of Economic Studies 66(1), 3-21.

[53] Mookherjee, D. (1984), "Optimal incentive schemes with many agents," Review of Economic Studies 51, 433-446.

[54] Radner, R. (1981), "Monitoring cooperative agreements in a repeated principal-agent relationship," Econometrica 49(5), 1127-1148.

[55] Riley J. G. (1985), "Competition with hidden knowledge," Journal of Political Economy $93,958-976$.

[56] Rob, R. and P. Zemsky (1999), "Cooperation, corporate culture and incentive intensity," CARESS Working Paper 97-09.

[57] Rogerson, W. (1985), "The first order approach to the principal-agent problems," Econometrica 53(6), 1357-1368.

[58] Rotemberg, J. (1994), "Human relations in the workplace," Journal of Political Economy $102(4), 684-717$.

[59] Ruef, M., H. E. Aldrich and N. M. Carter (2003), "The structure of founding teams: homophily, strong ties, and isolation among U. S. entrepreneurs," American Sociological Review 68 (2), 195-222.

[60] Ting, M. M. (2003), "A strategic theory of bureaucratic redundancy," American Journal of Political Science 47(2), 274-292.

[61] Waldstrom, C. (2001), "Informal networks in organizations - A literature review," Working Paper Aarhus School of Business 2001-2.

[62] Walker, C. and K. Dooley (1999), "The stability of self-organized rule-following work teams," Computational and Mathematical Organization Theory 5(1), 5-30.

[63] Winter, E. (2004), "Incentives and discriminations," American Economic Review 94, 764-773.

\section{A Appendix}

The following results will be used in some of the proofs. Denote by $\mathbf{G}$ the adjacency matrix of a network $g$ with undirected and simple graph; $\lambda_{G}$ its eigenvalues and $\bar{\lambda}_{G}=\max \left\{\lambda_{G}\right\}$. Brouwer and Haemers (2003) (BH): (i) For any $\mathbf{G}$, all $\lambda_{G}$ are real. (ii) If $\mathbf{G}$ is not connected, with separate connected components $\mathbf{G}_{\rho}$, the spectrum (the set of eigenvalues) of $\mathbf{G}$ is the union of the spectra of $\mathbf{G}_{\rho}$. (iii) Each connected $\mathbf{G}$ has an unique $\bar{\lambda}_{G}>0$, with $\left|\lambda_{G}\right|<\bar{\lambda}_{G}$. The function $\bar{\lambda}_{G}(\mathbf{G})$ decreases when vertices or links are removed from $g$. (iv) 
From (ii) and (iii) it follows that if $\mathbf{G}$ is not connected, there exists $\bar{\lambda}_{G}>0$ that is equal to the maximum eigenvalue of the component of maximum size (with more nodes) and more densely connected (with more links). If there are only one such large component, $\bar{\lambda}_{G}$ is unique. (v) G is irreducible (or indecomposable) if and only if the graph is connected. (vi) If $\mathbf{G}$ is irreducible (or indecomposable) then $k_{\min }<\bar{k}<\bar{\lambda}_{G}<k_{\max }$, where $k_{\min }, \bar{k}, k_{\max }$ are the minimum, average and maximum connectivity (or degree); in particular if $g$ is a $k$-regular graph, $\bar{\lambda}_{G}=k$. (vii) If $\mathbf{G}$ is not connected, $\bar{k} \leq \bar{\lambda}_{G} \leq k_{\max }$, and if $\bar{\lambda}_{G}=k_{\max }$ we only know that $\mathbf{G}$ has a component $k$-regular, although there may be other non-regular components. Debreu and Herstein (1953) (DH): (viii) For any $\mathbf{G},(s \mathbf{I}-\mathbf{G})^{-1} \geqq 0$ $\Longleftrightarrow s>\bar{\lambda}_{G}$ (Theorem III*); if $\mathbf{G}$ is indecomposable (i.e. $g$ is connected), $(s \mathbf{I}-\mathbf{G})^{-1}>0$ $\Longleftrightarrow s>\bar{\lambda}_{G}$ (Theorem III). (ix) For any matrix $\mathbf{A} \geqq 0$ indecomposable, its maximum eigenvalue increases when any element of $\mathbf{A}$ increases (Theorem I); if $\mathbf{A} \geqq 0$ is square, its maximum eigenvalue doesn't decrease when an element of $\mathbf{A}$ increases (Theorem I*).

\section{Proof of Proposition 1}

The principal's problem is:

$$
\max _{\left\{a_{i}, w_{i}\right\}_{i \in N}} E U_{P}=\sum_{i \in N}\left[\left(1+\beta \sum_{j \in N_{i}(g)} a_{j}\right) a_{i}-w_{i}\right], \text { s.t. } w_{i}-\frac{a_{i}^{2}}{2} \geq 0, i=1, \ldots, n
$$

Denote by $\mu_{i}$ the Lagrangian multiplier associated to each individual's participation constraint. From first order conditions it follows that for $i=1,2, \ldots, n$ and $\mu_{i}=1, w_{i}=\frac{a_{i}^{2}}{2}$. Solve the problem $\max _{\left\{a_{i}\right\}_{i \in N}} E U_{P}=\sum_{i \in N}\left[\left(1+\beta \sum_{j \in N_{i}(g)} a_{j}\right) a_{i}-\frac{a_{i}^{2}}{2}\right]$, which in turn implies that the solution (if it exists) is efficient. First order conditions are $1+\beta \sum_{j \in N_{i}(g)} a_{j}+$ $\sum_{j \in N_{i}(g)} \beta a_{j}-a_{i}=0 \Longrightarrow a_{i}=1+2 \beta \sum_{j \in N_{i}(g)} a_{j}$. Rewrite the $n$ equations in matrix form: $(\mathbf{I}-2 \beta \mathbf{G}) \overrightarrow{\mathbf{a}}=\overrightarrow{\mathbf{1}}$. By DH, Theorem III*,$(\mathbf{I}-2 \beta \mathbf{G})^{-1} \geqq 0 \Longleftrightarrow 1>2 \beta \bar{\lambda}_{G}$. Under A1, the candidate is:

$$
\overrightarrow{\mathbf{a}}^{*}=(\mathbf{I}-2 \beta \mathbf{G})^{-1} \overrightarrow{\mathbf{1}}
$$

Second order conditions. The matrix of second order derivatives $\mathbf{H}$ (of the relaxed problem) has elements $\frac{\partial^{2} U_{P}}{\partial a_{i}^{2}}=-1 \forall i i$ and $\frac{\partial^{2} U_{P}}{\partial a_{i} \partial a_{j}}=2 \beta$ if $j \neq i, j \in N_{i}(g), \frac{\partial^{2} U_{P}}{\partial a_{i} \partial a_{j}}=0$ otherwise. In compact form $\mathbf{H}=-(\mathbf{I}-2 \beta \mathbf{G})$. Therefore $\overrightarrow{\mathbf{a}}^{*}=\arg \max E U_{p} \Longleftrightarrow \mathbf{H}$ is negative definite $\Longleftrightarrow$ all its eigenvalues $\lambda_{H}$ are negative $\left(\lambda_{H}<0\right) . \lambda_{H}=(-1)\left(1-2 \beta \lambda_{G}\right)$ for all the eigenvalues of $\mathbf{G}, \lambda_{G}$. If $\lambda_{G} \leq 0 \Longrightarrow \lambda_{H}<0$. If $\lambda_{G}>0$ under A1 $2 \beta \lambda_{G} \leq$ $2 \beta \bar{\lambda}_{G}<1 \Longrightarrow \lambda_{H}<0 .{ }^{33}$ To obtain $E U_{P}^{*}$ note that in matrix form $E U_{P}=(\overrightarrow{\mathbf{1}}+$ $\beta \mathbf{G} \overrightarrow{\mathbf{a}})^{T} \overrightarrow{\mathbf{a}}-\frac{1}{2} \overrightarrow{\mathbf{a}}^{T} \overrightarrow{\mathbf{a}}=\overrightarrow{\mathbf{1}}^{T} \overrightarrow{\mathbf{a}}-\frac{1}{2} \overrightarrow{\mathbf{a}}^{T}(\mathbf{I}-2 \beta \mathbf{G}) \overrightarrow{\mathbf{a}}$

Finally, from $3,(\mathbf{I}-2 \beta \mathbf{G}) \frac{\partial \overrightarrow{\mathbf{a}}^{*}}{\partial \beta}-2 \mathbf{G} \overrightarrow{\mathbf{a}}^{*}=\overrightarrow{\mathbf{0}} \Longrightarrow \frac{\partial \overrightarrow{\mathbf{a}}^{*}}{\partial \beta}=2(\mathbf{I}-2 \beta \mathbf{G})^{-1} \mathbf{G}(\mathbf{I}-2 \beta \mathbf{G})^{-1} \overrightarrow{\mathbf{1}} \geqq$ $0 ; \frac{\partial^{2} \overrightarrow{\mathbf{a}}^{*}}{\partial \beta^{2}}=8(\mathbf{I}-2 \beta \mathbf{G})^{-1} \mathbf{G}(\mathbf{I}-2 \beta \mathbf{G})^{-1} \mathbf{G}(\mathbf{I}-2 \beta \mathbf{G})^{-1} \overrightarrow{\mathbf{1}} \geqq 0 ;$ and as $E U_{P}^{*}=\frac{1}{2} \overrightarrow{\mathbf{1}}^{T} \overrightarrow{\mathbf{a}}^{*} \Longrightarrow$ $\frac{\partial E U_{P}^{*}}{\partial \beta}>0$ and $\frac{\partial^{2} E U_{P}^{*}}{\partial \beta^{2}}>0 .^{3}$

\footnotetext{
${ }^{33}$ The set of eigenvalues of $(\mathbf{I}-2 \beta \mathbf{G})$ is $\left\{\left(1-2 \beta \lambda_{G}\right)\right\}$ where $\lambda_{G}$ are the eigenvalues of $\mathbf{G}$. Then, by eigenvalues' properties, as $\mathbf{H}=-(\mathbf{I}-2 \beta \mathbf{G}), \lambda_{H}=(-1)\left(1-2 \beta \lambda_{G}\right)$.

$34 \frac{\partial \overrightarrow{\mathbf{a}}^{*}}{\partial \beta}\left(\frac{\partial^{2} \overrightarrow{\mathbf{a}}^{*}}{\partial \beta^{2}}\right)$ is the $n \times 1$ vector of first (second) derivatives. If any agent $i$ is isolated, $a_{i}^{*}=1$, thus $\frac{\partial a_{i}^{*}}{\partial \beta}=0$ and $\frac{\partial^{2} a_{i}^{*}}{\partial \beta^{2}}=0$, otherwise the effort is a strictly increasing and strictly convex function of $\beta$. It
} 


\section{Proof of Proposition 2}

Denote by $\overrightarrow{\mathbf{d}}$ the $n \times 1$ vector of marginal incentives $\delta_{i}$ and $\mathbf{D}$ a $n$ diagonal matrix s.t. $\mathbf{D}=\operatorname{diag}(\overrightarrow{\mathbf{d}})$. Consider case (i), $w_{i}=\gamma_{i}+\delta_{i} y_{i}$. The payoffs of the induced network subgame are: $E U_{i}=\gamma_{i}+\delta_{i}\left(1+\beta \sum_{j \in N_{i}(g)} a_{j}\right) a_{i}-\frac{a_{i}^{2}}{2}$. Best response functions are: $\widehat{a}_{i}=$ $\delta_{i}\left(1+\beta \sum_{j \in N_{i}(g)} \widehat{a}_{j}\right) ;$ in matrix form:

$$
\overrightarrow{\mathbf{a}}=\overrightarrow{\mathbf{d}}+\beta \mathbf{D G} \overrightarrow{\mathbf{a}}
$$

Under case (ii), $w_{i}=\gamma_{i}+\delta_{i}\left(y_{i}+\sum_{j \in N_{i}(g)} y_{j}\right)$, payoffs are: $E U_{i}=\gamma_{i}+\delta_{i}\left[\left(1+\beta \sum_{j \in N_{i}(g)} a_{j}\right) a_{i}+\right.$ $\left.\sum_{j \in N_{i}(g)}\left(1+\beta \sum_{s \in N_{j}(g)} a_{s}\right) a_{j}\right]-\frac{a_{i}^{2}}{2}$. Best response functions are: $\widehat{a}_{i}=\delta_{i}\left(1+2 \beta \sum_{j \in N_{i}(g)} \widehat{a}_{j}\right)$; and the system is:

$$
\overrightarrow{\mathbf{a}}=\overrightarrow{\mathbf{d}}+2 \beta \mathbf{D G} \overrightarrow{\mathbf{a}}
$$

Since contracts can be personalized in both cases, the principal is able to extract to each agent her expected surplus. Therefore, in case (i): $\gamma_{i}=-\delta_{i} \widehat{x}_{i}+\frac{\widehat{a}_{i}^{2}}{2}$; and case (ii): $\gamma_{i}=-\delta_{i}\left(\widehat{x}_{i}+\sum_{j \in N_{i}(g)} \widehat{x}_{j}\right)+\frac{\widehat{a}_{i}^{2}}{2}$. All agents participate and in expected terms the allocation of total output is feasible. Given these fixed terms, in both cases $E U_{P}=\sum_{i \in N}\left(x_{i}-w_{i}\right)=$ $\sum_{i \in N}\left(x_{i}-\frac{a_{i}^{2}}{2}\right)$. We know that first best efforts $a_{i}^{*}$ maximize this function. It follows that if (i) $\delta_{i}=\frac{1+2 \beta \sum_{j \in N_{i}} \widehat{a}_{j}}{1+\beta \sum_{j \in N_{i}} \widehat{a}_{j}}=1+\frac{\beta \sum_{j \in N_{i}} \widehat{a}_{j}}{1+\beta \sum_{j \in N_{i}} \widehat{a}_{j}}$, the RHS of $(4) \Longrightarrow \overrightarrow{\mathbf{d}}+\beta \mathbf{D G} \overrightarrow{\mathbf{a}}=\overrightarrow{\mathbf{1}}+2 \beta \mathbf{G} \overrightarrow{\mathbf{a}} \Longrightarrow$ $\overrightarrow{\widehat{\mathbf{a}}}=\overrightarrow{\mathbf{1}}+2 \beta \mathbf{G} \overrightarrow{\widehat{\mathbf{a}}} \Longrightarrow \overrightarrow{\widehat{\mathbf{a}}}=\overrightarrow{\mathbf{a}}^{*}$ as eq. (4) becomes eq. (3); and in case (ii) $\delta_{i}=$ $1 \Longrightarrow \overrightarrow{\mathbf{a}}=\overrightarrow{\mathbf{1}}+2 \beta \mathbf{G} \overrightarrow{\mathbf{a}} \Longrightarrow \overrightarrow{\mathbf{a}}=\overrightarrow{\mathbf{a}}^{*}$ as eq. (5) becomes eq. (3). In both cases $\widehat{a}_{i}=a_{i}^{*} \Longrightarrow E U_{P}=E U_{P}^{*}$. Note that under $\mathrm{A} 1$ the equilibria in the effort subgames are interior and unique

\section{Proof of Proposition 3}

Suppose that the principal induce agents of different types to exert different levels of efforts. Without loss of generality, assume they are increasing in type; i.e. $\widehat{a}_{t} \geq \widehat{a}_{t^{\prime}} \Longleftrightarrow$ $t \geq t^{\prime}$. Then all agents participate if $w\left(\widehat{a}_{t}\right) \geq w\left(\widehat{a}_{t^{\prime}}\right)$. If the principal extracts all the surplus to each agent, for any type $t, w\left(\widehat{a}_{t}\right)=\frac{\widehat{a}_{t}^{2}}{2}$. Agents do no differ with respect to cost functions, therefore given this set of wages $\left(\left\{w\left(\widehat{a}_{t}\right), \widehat{a}_{t}\right\}_{t=1, \ldots . r}\right)$, all agents are indifferent among any of them. ${ }^{35}$ Any combination is a priori equally likely, hence the principal cannot induce one particular effort profile and in expectation, anticipates that any agent chooses the same

follows that $E U_{P}^{*}=\frac{1}{2} \sum_{i \in N} a_{i}^{*}$ is also strictly increasing and convex wrt $\beta$ unless all agents are isolated.

${ }^{35} \mathrm{As}$ is standard in this literature, incentive compatible contracts can be such that some types are indifferent between two contracts, but at least one type must strictly prefer the contract featured for her. Thus for example, if there are two types, high and low, the high type's contract leaves high type indifferent between that contract and the contract offered to the low type; but the low type has strictly no incentives to imitate high type. 
effort level. On the other hand, following standard arguments, suppose that the principal pays informational rents to agents of high types. In this case, because agents do not differ with respect to their cost functions, they all would choose the same contract that leaves agents with the higher informational rent. Wages depending only on efforts are not incentive compatible. The best the principal can do is to offer a common wage in exchange for the same level of effort to all agents and extract all the surplus: $w=\frac{a^{2}}{2}$. The principal's relaxed problem would be $\max _{a} E U_{P}=E\left\{\sum_{i \in N}\left[\left(1+\beta k_{i} a\right) a+\varepsilon_{i}-\frac{a^{2}}{2}\right]\right\}=n\left[(1+\beta \bar{k} a) a-\frac{a^{2}}{2}\right]$, where $k_{i}(g)$ is the number of neighbors $i$ has (degree or connectivity) and $\bar{k}$ is the average connectivity of network $g .{ }^{36}$ Optimal effort would be (solve FOC): $\widetilde{a}=\frac{1}{1-2 \beta \bar{k}}$. Under A1, $\widetilde{a}>0$ because $\bar{k} \leq \bar{\lambda}_{G}$ (by BH). Second order condition is $\frac{\partial^{2} E U_{P}}{\partial a^{2}}=2 \beta \bar{k}>0$. Given $\widetilde{a}, \widetilde{E U}_{P}=n \frac{1}{2} \frac{1}{1-2 \beta \bar{k}} \cdot \widetilde{E U}_{P}$ is obtained maximizing the same function as in the first best situation plus the constraint $a_{i}=a \forall i \in N$, therefore, $\widetilde{E U}_{P} \leq E U_{P}^{*}$ and only if the network is $k$-regular $\Longrightarrow \bar{k}=k \Longrightarrow \widetilde{a}=\frac{1}{1-2 \beta k}=a^{*}$ and $\widetilde{E U}_{P}=E U_{P}^{*}$, otherwise $\widetilde{E U}_{P}<E U_{P}^{*}$.

\section{Proof of Proposition 4}

Given the set of contracts, each agent $i$ of type $t$ participates and chooses contract $c_{t}$ if $\gamma_{t}+\delta_{t} x_{t(i)}^{*}-\frac{\left(a_{t(i)}^{*}\right)^{2}}{2} \geq 0$ and $\gamma_{t}+\delta_{t} x_{t(i)}^{*}-\frac{\left(a_{t(i)}^{*}\right)^{2}}{2} \geq \gamma_{t^{\prime}}+\delta_{t^{\prime}} \widetilde{x}_{t(i) \rightarrow t^{\prime}}-\frac{\left(a_{t^{\prime}(i)}^{*}\right)^{2}}{2}, \forall t^{\prime} \neq t$. As first best efforts are proportional to Bonacich centralities, $a_{t}^{*} \geq a_{t^{\prime}}^{*}$ and $x_{t}^{*} \geq x_{t^{\prime}}^{*} \Longleftrightarrow t \geq t^{\prime}$. For any agent $i$ of type $t, x_{t(i)}^{*}=x_{t}^{*}=\left(1+\beta \sum_{j \in N_{i}(g)} a_{t^{\prime}(j)}^{*}\right) a_{t(i)}^{*}$. For simplicity, denote by $\Phi_{t}^{*}=\left(1+\beta \sum_{j \in N_{i}(g)} a_{t^{\prime}(j)}^{*}\right)$, the factor that depends on neighbors' first best efforts, thus $x_{t}^{*}=\Phi_{t}^{*} a_{t}^{*}$. Note that $\Phi_{t}^{*} \geq \Phi_{t^{\prime}}^{*} \Longleftrightarrow t \geq t^{\prime}$; and that when any agent of type $t$ deviates unilaterally to another type $t^{\prime}, \widetilde{x}_{t \rightarrow t^{\prime}}=\Phi_{t}^{*} a_{t^{\prime}}^{*}$ as the rest of agents are choosing the right contract. $^{37}$ Now consider the contracts proposed. Assume that $\delta_{t}=\varepsilon>0$. We show that the relevant constraints are the local downward incentive constraints (LDIC) and the participation constraint of type 1. Sufficiency of local incentive compatibility conditions: Consider three types $t+1, t, t-1$. From the set of IC constraints, the following relations should hold in equilibrium:

$$
\begin{aligned}
\varepsilon \Phi_{t}^{*} & \left(a_{t}^{*}-a_{t-1}^{*}\right)-\frac{\left(a_{t}^{*}\right)^{2}-\left(a_{t-1}^{*}\right)^{2}}{2} \geq \gamma_{t-1}-\gamma_{t} \\
& \geq \varepsilon \Phi_{t-1}^{*}\left(a_{t}^{*}-a_{t-1}^{*}\right)-\frac{\left(a_{t}^{*}\right)^{2}-\left(a_{t-1}^{*}\right)^{2}}{2} \\
\varepsilon \Phi_{t+1}^{*} & \left(a_{t+1}^{*}-a_{t}^{*}\right)-\frac{\left(a_{t+1}^{*}\right)^{2}-\left(a_{t}^{*}\right)^{2}}{2} \geq \gamma_{t}-\gamma_{t+1} \\
& \geq \varepsilon \Phi_{t}^{*}\left(a_{t+1}^{*}-a_{t}^{*}\right)-\frac{\left(a_{t+1}^{*}\right)^{2}-\left(a_{t}^{*}\right)^{2}}{2} \\
\varepsilon \Phi_{t+1}^{*} & \left(a_{t+1}^{*}-a_{t-1}^{*}\right)-\frac{\left(a_{t+1}^{*}\right)^{2}-\left(a_{t-1}^{*}\right)^{2}}{2} \geq \gamma_{t-1}-\gamma_{t+1} \\
& \geq \varepsilon \Phi_{t-1}^{*}\left(a_{t+1}^{*}-a_{t-1}^{*}\right)-\frac{\left(a_{t+1}^{*}\right)^{2}-\left(a_{t-1}^{*}\right)^{2}}{2}
\end{aligned}
$$

\footnotetext{
${ }^{36}$ Strictly speaking, this average takes into account all the possible positions an agent would have given a particular network $g$; since any of these isomorphic structures arises with equal probability, it turns out that $\bar{k}$ can be directly calculated from any typical $g$ as the mean $\bar{k}=\frac{\sum_{i \in N} k_{i}(g)}{n}$

${ }^{37}$ For simplicity we drop the subindex $i$.
} 
It is easy to see that the three relations define non-empty ranges for the differences of fixed terms. (6) and (7) are local incentive compatibility conditions. Suppose that both are satisfied and sum them:

$$
\begin{aligned}
& \varepsilon \Phi_{t}^{*}\left(a_{t}^{*}-a_{t-1}^{*}\right)+ \varepsilon \Phi_{t+1}^{*}\left(a_{t+1}^{*}-a_{t}^{*}\right)-\frac{\left(a_{t+1}^{*}\right)^{2}-\left(a_{t-1}^{*}\right)^{2}}{2} \\
& \geq \gamma_{t-1}-\gamma_{t+1} \\
& \geq \varepsilon \Phi_{t-1}^{*}\left(a_{t}^{*}-a_{t-1}^{*}\right)+\varepsilon \Phi_{t}^{*}\left(a_{t+1}^{*}-a_{t}^{*}\right)-\frac{\left(a_{t+1}^{*}\right)^{2}-\left(a_{t-1}^{*}\right)^{2}}{2}
\end{aligned}
$$

Compare (9) and (8). LHS (8) $\geq \operatorname{LHS}(9)$ :

$$
\begin{gathered}
{\left[\varepsilon \Phi_{t+1}^{*}\left(a_{t+1}^{*}-a_{t-1}^{*}\right)-\frac{\left(a_{t+1}^{*}\right)^{2}-\left(a_{t-1}^{*}\right)^{2}}{2}\right]} \\
-\left[\varepsilon \Phi_{t}^{*}\left(a_{t}^{*}-a_{t-1}^{*}\right)+\varepsilon \Phi_{t+1}^{*}\left(a_{t+1}^{*}-a_{t}^{*}\right)-\frac{\left(a_{t+1}^{*}\right)^{2}-\left(a_{t-1}^{*}\right)^{2}}{2}\right] \\
=\varepsilon\left(\Phi_{t+1}^{*}-\Phi_{t}^{*}\right)\left(a_{t}^{*}-a_{t-1}^{*}\right) \geq 0
\end{gathered}
$$

$\operatorname{RHS}(9) \geq \operatorname{RHS}(8)$ :

$$
\begin{gathered}
{\left[\varepsilon \Phi_{t-1}^{*}\left(a_{t}^{*}-a_{t-1}^{*}\right)+\varepsilon \Phi_{t}^{*}\left(a_{t+1}^{*}-a_{t}^{*}\right)-\frac{\left(a_{t+1}^{*}\right)^{2}-\left(a_{t-1}^{*}\right)^{2}}{2^{2}}\right]} \\
-\left[\varepsilon \Phi_{t-1}^{*}\left(a_{t+1}^{*}-a_{t-1}^{*}\right)-\frac{\left(a_{t+1}^{*}\right)^{2}-\left(a_{t-1}^{*}\right)^{2}}{2}\right] \\
=\varepsilon\left(\Phi_{t}^{*}-\Phi_{t-1}^{*}\right)\left(a_{t+1}^{*}-a_{t}^{*}\right)^{2} \geq 0
\end{gathered}
$$

Then both local IC imply the non local one. Local downward incentive constraints (LDIC) bind at the optimum: Consider all the LDIC's and assume that for some type $t$ it is not binding: $\gamma_{t}+\varepsilon \Phi_{t}^{*} a_{t}^{*}-\frac{\left(a_{t}^{*}\right)^{2}}{2}>\gamma_{t-1}+\varepsilon \Phi_{t}^{*} a_{t-1}^{*}-\frac{\left(a_{t-1}^{*}\right)^{2}}{2}$. Then, the principal can adapt the incentives' scheme by raising the absolute value of all fixed payments of types $t^{\prime} \geq t$ by the same amount so as to make the preceding constraint binding. This would leave unaffected all the other LDIC's while improving the maximand. Participation constraints and LUIC: Finally, note that if LDICs are binding on the equilibrium $E U_{t} \geq E U_{t-1}$, as $\gamma_{t}+\varepsilon \Phi_{t}^{*} a_{t}^{*}-$ $\frac{\left(a_{t}^{*}\right)^{2}}{2}=\gamma_{t-1}+\varepsilon \Phi_{t}^{*} a_{t-1}^{*}-\frac{\left(a_{t-1}^{*}\right)^{2}}{2} \geq \gamma_{t-1}+\varepsilon \Phi_{t-1}^{*} a_{t-1}^{*}-\frac{\left(a_{t-1}^{*}\right)^{2}}{2}$, hence the unique participation constraint that binds is that of type $1 \Longrightarrow \gamma_{1}=-\varepsilon \Phi_{1}^{*} a_{1}^{*}+\frac{\left(a_{1}^{*}\right)^{2}}{2}$. It also implies that local upward incentive constraints (LUIC) can be omitted. From LDIC $E U_{t}=E U_{t \rightarrow t-1}$, $\gamma_{t}=\gamma_{t-1}-\varepsilon \Phi_{t}^{*}\left(a_{t}^{*}-a_{t-1}^{*}\right)+\frac{\left(a_{t}^{*}\right)^{2}-\left(a_{t-1}^{*}\right)^{2}}{2}$. Replace in the LUIC $E U_{t-1}-E U_{t-1 \rightarrow t} \Longrightarrow$ $\gamma_{t-1}+\varepsilon \Phi_{t-1}^{*} a_{t-1}^{*}-\frac{\left(a_{t-1}^{*}\right)^{2}}{2}-\gamma_{t}-\varepsilon \Phi_{t-1}^{*} a_{t}^{*}+\frac{\left(a_{t}^{*}\right)^{2}}{2}=\varepsilon\left(\Phi_{t}^{*}-\Phi_{t-1}^{*}\right)\left(a_{t}^{*}-a_{t-1}^{*}\right) \geq 0$. For any $\varepsilon>0$, agents will participate and reveal their types. Now we show that the expected utility of the principal comes close to the first best for $\varepsilon$ very small. Denote by $n_{t}=|N(t)|$. Fixed terms can be written as: $\gamma_{1}=-\varepsilon \Phi_{1}^{*} a_{1}^{*}+\frac{\left(a_{1}^{*}\right)^{2}}{2} ; \gamma_{t}=-\varepsilon \sum_{\tau=1}^{t} \Phi_{\tau}^{*} a_{\tau}^{*}+\varepsilon \sum_{\tau=2}^{t} \Phi_{\tau}^{*} a_{\tau-1}^{*}+\frac{\left(a_{t}^{*}\right)^{2}}{2}$, $\forall t \geq 2$. The expected utility of type $t$ when selecting contract $c_{t}: \stackrel{\tau=2}{E} U_{1}=0 ; E U_{t}=$ $\gamma_{t}+\varepsilon \Phi_{t}^{*} a_{t}^{*}-\frac{\left(a_{t}^{*}\right)^{2}}{2}=\varepsilon \sum_{\tau=2}^{t} a_{\tau-1}^{*}\left(\Phi_{\tau}^{*}-\Phi_{\tau-1}^{*}\right)>0, \forall t \geq 2$. Note that $E U_{t}$ is the informational rent that the principal pays to agents of type $t$. The principal's expected profit: 


$$
\begin{gathered}
E U_{P, A I}=\sum_{t=1}^{r} n_{t}\left(x_{t}^{*}(1-\varepsilon)-\gamma_{t}\right) \\
=\underbrace{\sum_{t=1}^{r} n_{t}\left(x_{t}^{*}-\frac{\left(a_{t}^{*}\right)^{2}}{2}\right)}_{E U_{P}^{*}}-\underbrace{\varepsilon \sum_{t=1}^{r} n_{t} \sum_{\tau=2}^{t} a_{\tau-1}^{*}\left(\Phi_{\tau}^{*}-\Phi_{\tau-1}^{*}\right)}_{\text {rents }}
\end{gathered}
$$

As for $\varepsilon$ very small contracts are still incentive compatible, the principal can obtain $E U_{P, A I} \approx E U_{P}^{*}$

\section{Proof of Proposition 5}

Assume any particular network $g$, choose $\beta \in\left(0, \frac{1}{2 \bar{\lambda}_{G}}\right)$ and consider a particular profile $\overrightarrow{\mathbf{d}}=\left\{\delta_{i}\right\}_{i \in N}$ chosen by agents in the previous stage. Provided that all participate, the expected payoffs of the induced effort subgame are: ${ }^{38}$

$$
E U_{i}=\gamma_{i}+\delta_{i}\left(1+\beta \sum_{j \in N_{i}(g)} a_{j}\right) a_{i}-\frac{a_{i}^{2}}{2}
$$

Agents' strategies are mappings from $\mathbb{R}_{+}^{n-1} \rightarrow \mathbb{R}_{+}$. That is, for any given profile of efforts chosen by agents in $N \backslash\{i\}$, individual $i$ chooses her effort $a_{i}$. The Nash equilibrium of the effort subgame is an effort profile $\left(\widehat{a}_{i}\right)_{i \in N}$ such that for $\forall i: E U_{i}\left(\widehat{a}_{i}, \widehat{a}_{-i}\right) \geq E U_{i}\left(a_{i}, \widehat{a}_{-i}\right)$ $\forall a_{i} \neq \widehat{a}_{i} \Longrightarrow \widehat{a}_{i} \in \arg \max _{a_{i}} E U_{i}\left(a_{i}, \widehat{a}_{-i}\right)$ (where as usual $\widehat{a}_{-i}$ stands for the equilibrium choices of agents in $N \backslash\{i\})$. Best response functions in matrix form are $(\mathbf{I}-\beta \mathbf{D G}) \overrightarrow{\mathbf{a}}=\overrightarrow{\mathbf{d}}$. The existence and uniqueness of the NE of the effort network subgame requires that $\mathbf{I}-\beta \mathbf{D G}$ be inverse-positive, that requires (see the proof of Prop. 1) that $1>\beta \max \left\{\lambda_{D G}\right\}$, where $\lambda_{D G}$ range over all the eigenvalues of DG. However, $\max \left\{\lambda_{D G}\right\}$ doesn't decrease when any element of DG increases (DH Theorem I*). Hence consider another profile $\overrightarrow{\widetilde{\mathbf{d}}} \supsetneqq \overrightarrow{\mathbf{d}}$ where at least one of its elements increases (i.e. simply assume that agent $j$ changes its choice $\widetilde{\delta}_{j}>$ $\delta_{j}$ ). Then, all the elements of row $j$ of $\widetilde{\mathbf{D}} \mathbf{G}$ have increased and $\max \left\{\lambda_{\widetilde{D} G}\right\} \geq \max \left\{\lambda_{D G}\right\}$. It follows that if all agents choose the same contract, namely the one with the maximum $\bar{\delta}$, $\max \left\{\lambda_{\bar{D} G}\right\} \geq \max \left\{\lambda_{D G}\right\}$ for any other $\overrightarrow{\mathbf{d}}$. If $\delta_{i}=\bar{\delta} \forall i \in N, \mathbf{I}-\beta \mathbf{D G}=\mathbf{I}-\beta \bar{\delta} \mathbf{G}$, and the $\mathrm{DH}$ condition would imply that $1>\beta \overline{\delta \lambda}_{G}$.

Now, consider two different profiles $\overrightarrow{\mathbf{d}}$ and $\overrightarrow{\widetilde{\mathbf{d}}}$; recall that assumption $\bar{\delta} \in\left(0, \frac{1}{\beta \bar{\lambda}_{G}}\right)$ is sufficient to have the two interior solutions. ${ }^{39}$ Define $\overrightarrow{\widetilde{\mathbf{d}}}=\overrightarrow{\mathbf{d}}+\overrightarrow{\mathbf{z}}: z_{i}=\widetilde{\delta}_{i}-\delta_{i} \geq 0$ with at least one inequality strict and $\widetilde{\mathbf{D}}=\mathbf{D}+\mathbf{Z}: \operatorname{diag}(\mathbf{Z})=\overrightarrow{\mathbf{z}}$. Use $(\mathbf{I}-\beta(\mathbf{D}+\mathbf{Z}) \mathbf{G}) \overrightarrow{\overrightarrow{\mathbf{a}}}=$ $\overrightarrow{\mathbf{d}}+\overrightarrow{\mathbf{z}}$ and $(\mathbf{I}-\beta \mathbf{D G}) \overrightarrow{\mathbf{a}}=\overrightarrow{\mathbf{d}}$ :

$$
\begin{gathered}
(\mathbf{I}-\beta(\mathbf{D}+\mathbf{Z}) \mathbf{G}) \overrightarrow{\overrightarrow{\mathbf{a}}}=\overrightarrow{\mathbf{d}}+\overrightarrow{\mathbf{z}} \\
(\mathbf{I}-\beta \mathbf{D G}) \overrightarrow{\overrightarrow{\mathbf{a}}}-\beta \mathbf{Z G} \overrightarrow{\overrightarrow{\mathbf{a}}}=(\mathbf{I}-\beta \mathbf{D G}) \overrightarrow{\mathbf{a}}+\overrightarrow{\mathbf{z}} \\
\overrightarrow{\overrightarrow{\mathbf{a}}}-\overrightarrow{\mathbf{a}}=(\mathbf{I}-\beta \mathbf{D G})^{-1}(\overrightarrow{\mathbf{z}}+\beta \mathbf{Z G} \overrightarrow{\overrightarrow{\mathbf{a}}})>0 .
\end{gathered}
$$

\footnotetext{
${ }^{38}$ As we will see participation is modulated by fixed terms $\gamma_{i}$. It is obvious that for some combination of contracts chosen, some agent at the end would reject to participate. However, at this stage we do not need to care about that. Since $\gamma_{i}$ is a fixed term, it does not affect optimal effort choices under some particular profile $\overrightarrow{\mathbf{d}}$. Naturally, if under such profile some agent doesn't obtain her reservation utility, then such $\overrightarrow{\mathbf{d}}$ will not be part of the equilibrium of the full game.

${ }^{39}$ Both profiles belong to the same set offered by the principal, where $\bar{\delta}$ is the maximum of all $\delta_{t}$ available.
} 
Finally, given any profile $\overrightarrow{\mathbf{d}}$, use Cramer's rule to write down the equilibrium effort (of the correspondent subgame) of agent $i$. The denominator is $|\mathbf{I}-\beta \mathbf{D G}|{ }^{40}$ develop it by cofactors of row $i$ : then $|\mathbf{I}-\beta \mathbf{D G}|=A_{i i}-\delta_{i} \beta \sum_{j \in N_{i}} A_{i j}$, where $A_{i i}=(-1)^{2 i} M_{i i}$ and $A_{i j}=(-1)^{i+j} M_{i j} ; M_{i i}$ is the principal minor $i$ and $M_{i j}$ are the complementary minors of elements $i j$. Neither $M_{i i}$ nor $M_{i j}$ 's depend on $\delta_{i}$. Since $\mathbf{I}-\beta \mathbf{D G}$ is inverse positive, $|\mathbf{I}-\beta \mathbf{D G}|>0$ and $(\mathbf{I}-\beta \mathbf{D G})^{-1}=\frac{1}{|\mathbf{I}-\beta \mathbf{D G}|} \operatorname{Adj}(\mathbf{I}-\beta \mathbf{D G}) \Longrightarrow A_{i i}$ and all $A_{i j}$ are positive $\left(A_{i i}\right.$ and $A_{i j}$ are elements of $\left.\operatorname{Adj}(\mathbf{I}-\beta \mathbf{D G})\right)$. Next, develop the numerator by cofactors of column $i \Longrightarrow \delta_{i} A_{i i}+\sum_{j \neq i} \delta_{j} A_{j i}$. Cofactors $A_{j i}$ and $A_{i j}$ are related as follows: $A_{j i}=\frac{\delta_{i}}{\delta_{j}} A_{i j}$. Replacing, we can write $\delta_{i} A_{i i}+\sum_{j \neq i} \delta_{j} A_{j i}=\delta_{i} A_{i i}+\sum_{j \neq i} \delta_{j} \frac{\delta_{i}}{\delta_{j}} A_{i j}=$ $\delta_{i}\left(A_{i i}+\sum_{j \neq i} A_{i j}\right)=\delta_{i}\left(A_{i i}+\sum_{j \in N_{i}} A_{i j}+\sum_{j \neq i: j \notin N_{i}} A_{i j}\right)$. (Note that none of these cofactors depend on $\delta_{i}$, and recall that $\left.A_{i j} \geq 0\right)$. It follows that the effort of agent $i$ for a given profile $\widetilde{\delta}_{-i}$ can be written as a continuous function of $\delta_{i}$ :

$$
\begin{gathered}
a_{i}\left(\delta_{i}, \widetilde{\delta}_{-i}\right)=\delta_{i} \frac{A_{i i}+\sum_{j \in N_{i}} A_{i j}+\sum_{j \neq i: j \notin N_{i}} A_{i j}}{A_{i i}-\delta_{i} \beta \sum_{j \in N_{i}} A_{i j}}, \\
\frac{\partial a_{i}\left(\delta_{i}, \widetilde{\delta}_{-i}\right)}{\partial \delta_{i}}>0 ; \frac{\partial^{2} a_{i}\left(\delta_{i} \widetilde{\delta}_{-i}\right)}{\partial \delta_{i}^{2}}>0 .
\end{gathered}
$$

\section{Proof of Proposition 6}

Given the common wage offered, $(\gamma, \delta)$, and assuming that all agents participate, the payoffs of the induced network subgame are: $E U_{i}=\gamma+\delta\left(1+\beta \sum_{j \in N_{i}} a_{j}\right) a_{i}-\frac{a_{i}^{2}}{2}$. Thus best response functions are $\delta\left(1+\beta \sum_{j \in N_{i}} a_{j}\right)-a_{i}=0 \Longrightarrow \widehat{a}_{i}=\delta\left(1+\beta \sum_{j \in N_{i}} \widehat{a}_{j}\right)$. Compare to first best efforts which satisfy $a_{i}^{*}=\left(1+2 \beta \sum_{j \in N_{i}} a_{j}^{*}\right)$. Trivially there is not $\delta$ such that $\widehat{a}_{i}=a_{i}^{*}$ for all $i$ simultaneously. On the other hand, for $\delta>0$, the equilibrium effort chosen by $i$ is proportional to her Bonacich centrality and satisfy $a_{i}^{2}=\delta x_{i} \Longrightarrow a_{i}>a_{j} \Longleftrightarrow x_{i}>x_{j}$. The principal is able to know in advance that given $\delta>0$, there will be some agent(s) with the minimum Bonacich centrality that will produce the lowest (i.e. type $t=1$ ). These are the agents from which the principal is able to extract all the surplus through $\gamma$ : $E U_{1}=\gamma+\delta \widehat{x}_{1}-\frac{\widehat{a}_{1}^{2}(\delta)}{2}=0 \Longrightarrow \gamma=\frac{\widehat{a}_{1}^{2}(\delta)}{2}-\delta \widehat{x}_{1}$, use $\widehat{x}_{1}=\frac{\widehat{a}_{1}^{2}}{\delta} \Longrightarrow \gamma=-\frac{\widehat{a}_{1}^{2}(\delta)}{2}$. The principal's problem is then to choose $\delta$ to maximize $(1-\delta) X(\delta)+n \frac{\widehat{a}_{1}^{2}(\delta)}{2}$, with $X(\delta)=$ $(\overrightarrow{\mathbf{1}}+\beta \mathbf{G} \overrightarrow{\widehat{\mathbf{a}}})^{T} \overrightarrow{\widehat{\mathbf{a}}}$, and $\overrightarrow{\widehat{\mathbf{a}}}=\delta(\mathbf{I}-\delta \beta \mathbf{G})^{-1} \overrightarrow{\mathbf{1}}$. First order condition is given by:

$$
-X(\delta)+(1-\delta) \frac{\partial X(\delta)}{\partial \delta}+n \widehat{a}_{1}(\delta) \frac{\partial \widehat{a}_{1}(\delta)}{\partial \delta}=0
$$

with $\frac{\partial X(\delta)}{\partial \delta}=(\overrightarrow{\mathbf{1}}+2 \beta G \overrightarrow{\widehat{\mathbf{a}}})^{T} \frac{\partial \overrightarrow{\mathbf{a}}}{\partial \delta}$; from which the self-consistency condition for $\widehat{\delta}$ is obtained. If the network is $k$-regular, in equilibrium all agents exert the same effort $\widehat{a}_{i}=$ $\widehat{a}=\frac{\delta}{1-\delta \beta k} ; X(\delta)=n(1+\beta k \widehat{a}) \widehat{a}$ and the principal extracts all the surplus to all agents, $\gamma=-\frac{\widehat{a}^{2}}{2}$. The optimal marginal participation is $\widehat{\delta}(k)=\frac{1}{1-\beta k} \Longrightarrow \widehat{a}=a^{*}$ and $E U_{P, S C}=$

\footnotetext{
${ }^{40}$ Matrix I $-\beta \mathbf{D G}$ has its diagonal elements equal to 1 , and any element $i j$ is equal to $-\beta \delta_{i}$ if $g_{i j}=1$ or zero if $g_{i j}=0$ - it is not symmetric since if $g_{i j}=g_{j i}=1$, element $j i$ will be equal to $-\beta \delta_{j}$.
} 
$E U_{P}^{*}=\frac{1}{2} \frac{n}{1-2 \beta k}$. Observation: the value of $\widehat{\delta}$ depends on the particular network and $\beta$, as it is shown in the text.

\section{Proof of Proposition 7}

Notation: $\widehat{a}_{t}=\widehat{a}_{t(i)}\left(\widehat{\delta}_{t}, \widehat{\delta}_{-i}\right)$, effort of agent of type $t$ on the equilibrium path. $\widetilde{a}_{t \rightarrow t^{\prime}}=$ $\widetilde{a}_{t(i)}\left(\delta_{t^{\prime}}, \widehat{\delta}_{-i}\right)$, effort when an agent of type $t$ deviates unilaterally and chooses contract $t^{\prime}$. In the first best, the ratio of marginal cost to marginal productivity $\frac{M C_{t}}{M P_{t}}$ of agents increases with type (footnotes 23,24 and notation of proof Prop. 4). By offering the menu of contracts, on the equilibrium path, $\widehat{\delta}_{t}=\frac{M C_{t}}{M P_{t}}$; therefore the principal will improve his profits with respect to the case of single contracts if he induces the same tendency of $\frac{M C_{t}}{M P_{t}}$ as in the first best and $\widehat{\delta}_{t} \geq \widehat{\delta}_{t^{\prime}} \Longleftrightarrow t \geq t^{\prime}$. Weakly increasing marginal incentives imply that equilibrium efforts are increasing in type. (Recall that even if marginal incentives are equal, efforts are increasing in type). By Prop. 5 the effort of any agent is (ceteris paribus) increasing and strictly convex in her marginal participation, hence any unilateral deviation yields higher effort when imitating higher types and viceversa. It follows that if $\widehat{\delta}_{t} \geq \widehat{\delta}_{t^{\prime}} \Longleftrightarrow t \geq t^{\prime}$, then $\widehat{\gamma}_{t^{\prime}} \geq \widehat{\gamma}_{t}$. Suppose not, $\widehat{\gamma}_{t^{\prime}}<\widehat{\gamma}_{t}$. Then $\widehat{\gamma}_{t^{\prime}}+\frac{\widehat{a}_{t^{\prime}}^{2}}{2}<\widehat{\gamma}_{t}+\frac{\widetilde{a}_{t^{\prime} \rightarrow t}^{2}}{2}$ since by Prop. $5 \frac{\widetilde{a}_{t^{\prime} \rightarrow t}^{2}}{2}>\frac{\widehat{a}_{t^{\prime}}^{2}}{2}$, i.e. agents would imitate higher types. We therefore assume that $\widehat{\delta}_{t} \geq \widehat{\delta}_{t^{\prime}} \Longleftrightarrow t \geq t^{\prime}$ (thus $\widehat{\gamma}_{t^{\prime}} \geq \widehat{\gamma}_{t}$ ) and that the other givens hold and follow the same steps as in the proof of Prop. 4. We show that the relevant constraints are such that any agent of type $t$ left indifferent between contract $t$ and $t-1$, provided that all participate. This minimizes the informational rents. Sufficiency of local incentive compatibility conditions: Consider three types $t+1, t, t-1$. From the set of IC constraints (use formulation (2') of the text), the following relations should hold in equilibrium:

$$
\begin{gathered}
\frac{\widehat{a}_{t}^{2}}{2}-\frac{\widetilde{a}_{t \rightarrow t-1}^{2}}{2} \geq \gamma_{t-1}-\gamma_{t} \geq \frac{\widetilde{a}_{t-1 \rightarrow t}^{2}}{2}-\frac{\widehat{a}_{t-1}^{2}}{2} \\
\frac{\widehat{a}_{t+1}^{2}}{2}-\frac{\widetilde{a}_{t+1 \rightarrow t}^{2}}{2} \geq \gamma_{t}-\gamma_{t+1} \geq \frac{\widetilde{a}_{t \rightarrow t+1}^{2}}{2}-\frac{\widehat{a}_{t}^{2}}{2} \\
\frac{\widehat{a}_{t+1}^{2}}{2}-\frac{\widetilde{a}_{t+1 \rightarrow t-1}^{2}}{2} \geq \gamma_{t-1}-\gamma_{t+1} \geq \frac{\widetilde{a}_{t-1 \rightarrow t+1}^{2}}{2}-\frac{\widehat{a}_{t}^{2}}{2}
\end{gathered}
$$

(10) and (11) are local incentive compatibility conditions. Suppose that both are satisfied and sum them:

$$
\begin{gathered}
\frac{\widehat{a}_{t}^{2}}{2}-\frac{\widetilde{a}_{t \rightarrow t-1}^{2}}{2}+\frac{\widehat{a}_{t+1}^{2}}{2}-\frac{\widetilde{a}_{t+1 \rightarrow t}^{2}}{2} \geq \gamma_{t-1}-\gamma_{t+1} \\
\frac{\widetilde{a}_{t-1 \rightarrow t}^{2}}{2}-\frac{\widehat{a}_{t-1}^{2}}{2}+\frac{\widetilde{a}_{t \rightarrow t+1}^{2}}{2}-\frac{\widehat{a}_{t}^{2}}{2}
\end{gathered}
$$

(13) $\Longrightarrow$ (12) if LHS (12) $\geq$ LHS (13) and RHS (13) $\geq$ RHS (12), which hold as LHS (12) - LHS (13) $=\frac{\widetilde{a}_{t+1 \rightarrow t}^{2}-\widetilde{a}_{t+1 \rightarrow t-1}^{2}}{2}-\frac{\widehat{a}_{t}^{2}-\widetilde{a}_{t \rightarrow t-1}^{2}}{2} \geq 0$ and RHS (13) - RHS (12) $=\frac{\widetilde{a}_{t \rightarrow t+1}^{2}-\widehat{a}_{t}^{2}}{2}-\frac{\widetilde{a}_{t-1 \rightarrow t+1}^{2}-\widetilde{a}_{t-1 \rightarrow t}^{2}}{2} \geq 0$. Then both local IC imply the non local one. Local downward incentive constraints (LDIC) bind at the optimum: Consider all the LDIC's and assume that for some type $t$ it is not binding: $\gamma_{t}+\frac{\widehat{a}_{t}^{2}}{2}>\gamma_{t-1}+\frac{\widetilde{a}_{t \rightarrow t-1}^{2}}{2}$. Then, the principal can adapt the incentives' scheme by raising all the absolute values of fixed payments of types $t^{\prime} \geq t$ by the same amount so as to make the preceding constraint binding. This 
would leave unaffected all the other LDIC's while reducing his cost, as fixed terms $\gamma_{t}$ are positive transfers that the principal receive from agents. Participation constraints and LUIC: If LDICs are binding, the unique participation constraint that binds if that of type 1 as $\gamma_{t}+\frac{\widehat{a}_{t}^{2}}{2}=\gamma_{t-1}+\frac{\widetilde{a}_{t \rightarrow t-1}^{2}}{2} \geq \gamma_{t-1}+\frac{\widehat{a}_{t-1}^{2}}{2} \Longrightarrow \gamma_{1}=-\frac{\widehat{a}_{1}^{2}}{2}$ and local upward incentive constraints (LUIC) can be omitted: $\gamma_{t}+\frac{\widehat{a}_{t}^{2}}{2}=\gamma_{t-1}+\frac{\widetilde{a}_{t \rightarrow t-1}^{2}}{2} \Longrightarrow \gamma_{t}=\gamma_{t-1}+\frac{\widetilde{a}_{t \rightarrow t-1}^{2}}{2}-\frac{\widehat{a}_{t}^{2}}{2}$. Replace in the LUIC: $\gamma_{t-1}+\frac{\widehat{a}_{t-1}^{2}}{2}-\left(\gamma_{t}+\frac{\widetilde{a}_{t-1 \rightarrow t}^{2}}{2}\right) \geq 0 \Longrightarrow \frac{\widehat{a}_{t}^{2}-\widetilde{a}_{t \rightarrow t-1}^{2}}{2}-\frac{\widetilde{a}_{t-1 \rightarrow t}^{2}-\widehat{a}_{t-1}^{2}}{2} \geq 0$. At last notice that the single contract is always a possible solution of the principal's problem. Therefore, $E \widehat{U}_{P, M C} \geq E \widehat{U}_{P, S C}$. On the other hand, if some separation occurs, then the principal pays informational rents and $E \widehat{U_{P, M C}}<E U_{P}^{*}$

\section{Networks}

(Labels in the graphs denote "type.")



Maximum eigenvalues, $\bar{\lambda}_{G}: \bar{\lambda}_{\text {star }}=(n-1)^{\frac{1}{2}} ; \bar{\lambda}_{I L 2 S}=\frac{1}{2}+\frac{(8 n-15)^{\frac{1}{2}}}{2} ; \bar{\lambda}_{I L 3 S}=1+(3 n-$ $8)^{\frac{1}{2}} ; \bar{\lambda}_{C P 1}=\frac{n-4}{8}+\left(\left(\frac{n-4}{8}\right)^{2}+3\right)^{\frac{1}{2}}, \frac{n}{4}$ integer; $\bar{\lambda}_{C P 2}=\frac{n-3}{6}+\left(\left(\frac{n-3}{6}\right)^{2}+2\right)^{\frac{1}{2}}, \frac{n}{3}$ integer; $\bar{\lambda}_{C P 3}=\frac{n-2}{4}+\left(\left(\frac{n-2}{4}\right)^{2}+1\right)^{\frac{1}{2}}, \frac{n}{2}$ integer; $\bar{\lambda}_{T j}=\left(\frac{n-1}{2}+\left(\left(\frac{n-1}{2}\right)^{2}-n_{1} n_{2}\right)^{\frac{1}{2}}\right)^{\frac{1}{2}}, j=1,2,3,4$, $n=n_{1}+n_{2}+2, n_{1}, n_{2}$ are "end" nodes, i.e. $n_{1}=|N(1)|, n_{2}=|N(2)| ; \bar{\lambda}_{E G j}=n_{c}-1$, $j=1,2,3, n_{c}$ : maximum component size; $\bar{\lambda}_{\text {line }}=-2 \cos \left(\pi \frac{n}{n+1}\right) ; \bar{\lambda}_{M L 1}=2.247$ (for $n=12) ; \bar{\lambda}_{M L 2}=2.7531$ (for $n=12$ ). 